\title{
Directions of Motion Fields are Hardly Ever Ambiguous
}

\author{
TOMAS BRODSKY, CORNELIA FERMÜLLER AND YIANNIS ALOIMONOS* \\ Computer Vision Laboratory, Center for Automation Research, Computer Science Department, and Institute for \\ Advanced Computer Studies, University of Maryland, College Park MD 20742-3275, USA \\ fer@cfar.umd.edu \\ brodsky@cfar.umd.edu \\ yiannis@cfar.umd.edu
}

Received November 11, 1995; Accepted June 30, 1996

\begin{abstract}
If instead of the full motion field, we consider only the direction of the motion field due to a rigid motion, what can we say about the three-dimensional motion information contained in it? This paper provides a geometric analysis of this question based solely on the constraint that the depth of the surfaces in view is positive. The motivation behind this analysis is to provide a theoretical foundation for image constraints employing only the sign of flow in various directions and justify their utilization for addressing 3D dynamic vision problems.

It is shown that, considering as the imaging surface the whole sphere, independently of the scene in view, two different rigid motions cannot give rise to the same directional motion field. If we restrict the image to half of a sphere (or an infinitely large image plane) two different rigid motions with instantaneous translational and rotational velocities $\left(\mathbf{t}_{1}, \boldsymbol{\omega}_{1}\right)$ and $\left(\mathbf{t}_{2}, \boldsymbol{\omega}_{2}\right)$ cannot give rise to the same directional motion field unless the plane through $\mathbf{t}_{1}$ and $\mathbf{t}_{2}$ is perpendicular to the plane through $\boldsymbol{\omega}_{1}$ and $\boldsymbol{\omega}_{2}$ (i.e., $\left(\mathbf{t}_{1} \times \mathbf{t}_{2}\right) \cdot\left(\boldsymbol{\omega}_{1} \times \boldsymbol{\omega}_{2}\right)=0$ ). In addition, in order to give practical significance to these uniqueness results for the case of a limited field of view, we also characterize the locations on the image where the motion vectors due to the different motions must have different directions.

If $\left(\boldsymbol{\omega}_{1} \times \boldsymbol{\omega}_{2}\right) \cdot\left(\mathbf{t}_{1} \times \mathbf{t}_{2}\right)=0$ and certain additional constraints are met, then the two rigid motions could produce motion fields with the same direction. For this to happen the depth of each corresponding surface has to be within a certain range, defined by a second and a third order surface. Similar more restrictive constraints are obtained for the case of multiple motions. Consequently, directions of motion fields are hardly ever ambiguous. A byproduct of the analysis is that full motion fields are never ambiguous with a half sphere as the imaging surface.
\end{abstract}

Keywords: qualitative vision, motion field, optic flow, egomotion

\section{Introduction and Motivation}

The basis of the majority of visual motion studies has been the motion field, i.e., the projection of the

\footnotetext{
*The support of the Advanced Research Projects Agency (ARPA Order No. 8459) and the U.S. Army Topographic Engineering Center under Contract DACA76-92-C-0009, the Office of Naval Research under Contract N00014-93-1-0257, National Science Foundation under Grant IRI-90-57934, and the Austrian "Fonds zur Förderung der wissenschaftlichen Forschung," project No. S 7003, is gratefully acknowledged.
}

velocities of $3 \mathrm{D}$ scene points on the image. Classical results on the uniqueness of motion fields (Horn, 1987; Maybank, 1993; Negahdaripour, 1989) as well as displacement fields (Longuet-Higgins, 1981; Spetsakis and Aloimonos, 1990; Tsai and Huang, 1984) have formed the foundation of most research on rigid motion analysis that addressed the 3D motion problem by first approximating the motion field through the optical flow and then interpreting the optical flow to obtain 3D motion and structure (Faugeras et al., 1987; Horn, 1990; Tistarelli and Sandini, 1992; Vieville and Faugeras, 1994). 
The difficulties involved in the estimation of optical flow have recently given rise to a small number of studies considering as input to the visual motion interpretation process some partial optical flow information. In particular the projection of the optical flow on the gradient direction, the so-called normal flow (Fermüller and Aloimonos, 1995; Negahdaripour and Horn, 1987), and the projections of the flow on different directions (Ancona and Poggio, 1995; Fermüller, 1995) have been utilized. In Fermüller (1995) constraints on the sign of the projection of the flow on various directions were presented. These constraints on the sign of the flow were derived using only the rigid motion model, with the only constraint on the scene being that the depth in view had to be positive at every point - the so-called "depth-positivity" constraint. In the sequel we are led naturally to the question of what these constraints, or more generally any constraint on the sign of the flow, can possibly tell us about three-dimensional motion and the structure of the scene in view. Thus we would like to investigate the amount of information in the sign of the projection of the flow. Since knowing the sign of the projection of a motion vector in all directions is equivalent to knowing the direction of the motion vector, our question amounts to studying the relationship between the directions of $2 \mathrm{D}$ motion vectors and $3 \mathrm{D}$ rigid motion.

We next state the well-known equation for rigid motion for the case of a spherical imaging surface. We describe the constraints and discuss the information exploited when using the full flow as opposed to the information employed when using only the direction of flow. As will be shown, whereas full flow allows for derivation of the direction of translation and the complete rotation, from the orientation of the flow only the direction of translation and the direction of rotation can be obtained.

The 2D motion field on the imaging surface is the projection of the 3D motion field of the scene points moving relative to that surface. Suppose the observer is moving rigidly with instantaneous translation $\mathbf{t}=$ $(U, V, W)$ and instantaneous rotation $\boldsymbol{\omega}=(\alpha, \beta, \gamma)$ (see Fig. 1). Each scene point $\mathbf{R}=(X, Y, Z)$ measured with respect to a coordinate system $O X Y Z$ fixed to the camera moves relative to the camera with velocity $\dot{\mathbf{R}}$, where

$$
\dot{\mathbf{R}}=-\mathbf{t}-\boldsymbol{\omega} \times \mathbf{R}
$$

If the center of projection is at the origin and the image is formed on a sphere with radius 1 , the relationship

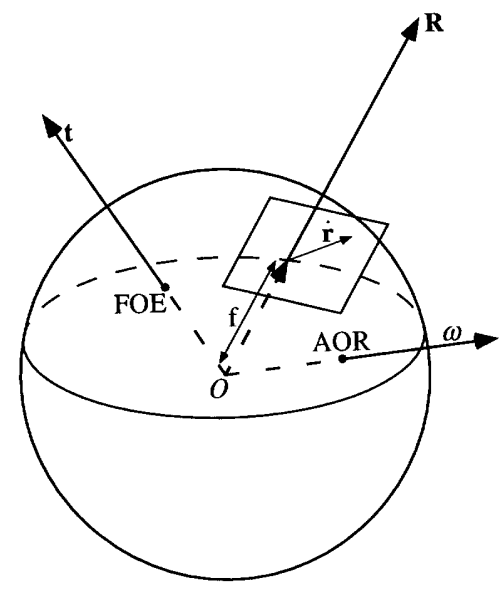

Figure 1. Image formation on a spherical retina under perspective projection.

between the image point $\mathbf{r}$ and the scene point $\mathbf{R}$ under perspective projection is

$$
\mathbf{r}=\frac{\mathbf{R}}{|\mathbf{R}|}
$$

with $|\mathbf{R}|$ being the norm of the vector $\mathbf{R}$.

If we now differentiate $\mathbf{r}$ with respect to time and substitute for $\dot{\mathbf{R}}$, we obtain the following equation for $\dot{\mathbf{r}}$ :

$$
\begin{aligned}
\dot{\mathbf{r}}=v_{\text {tr }}(\mathbf{r})+v_{\text {rot }}(\mathbf{r}) & =\frac{1}{|\mathbf{R}|}((\mathbf{t} \cdot \mathbf{r}) \mathbf{r}-\mathbf{t})-\boldsymbol{\omega} \times \mathbf{r} \\
& =-\frac{1}{|\mathbf{R}|}(\mathbf{r} \times(\mathbf{t} \times \mathbf{r}))-\boldsymbol{\omega} \times \mathbf{r}
\end{aligned}
$$

The first term $v_{\text {tr }}(\mathbf{r})=\frac{1}{|\mathbf{R}|}((\mathbf{t} \cdot \mathbf{r}) \mathbf{r}-\mathbf{t})$ corresponds to the translational component which depends on the depth $Z=|\mathbf{R}|$, the distance of $\mathbf{R}$ to the center of projection. The direction of $v_{\text {tr }}(\mathbf{r})$ is along great circles (longitudes) pointing away from the Focus of Expansion $(\mathbf{t})$ and towards the Focus of Contraction $(-\mathbf{t})$. The second term $v_{\text {rot }}(\mathbf{r})=-\boldsymbol{\omega} \times \mathbf{r}$ corresponds to the rotational component which is independent of depth. Its direction is along latitudes around the axis of rotation (counterclockwise around $\boldsymbol{\omega}$ and clockwise around $-\boldsymbol{\omega})$. See Figs. 2(a), (b), and (c) for translational, rotational, and general motion fields on the sphere.

As can be seen, without additional constraints there is an ambiguity in the computation of shape and translation. It is not possible to disentangle the effects of $\mathbf{t}$ and $|\mathbf{R}|$, and thus we can only derive the direction of 


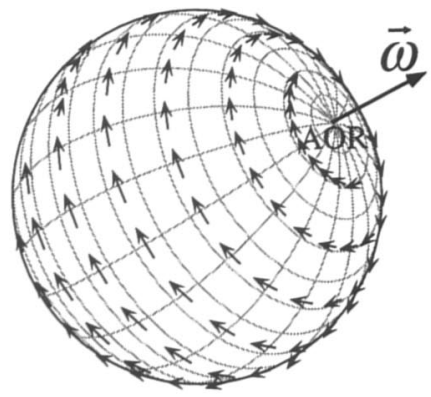

(a)

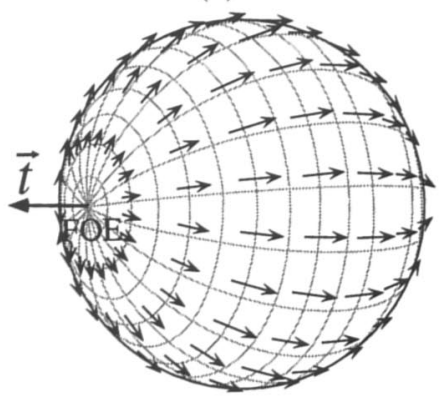

(b)

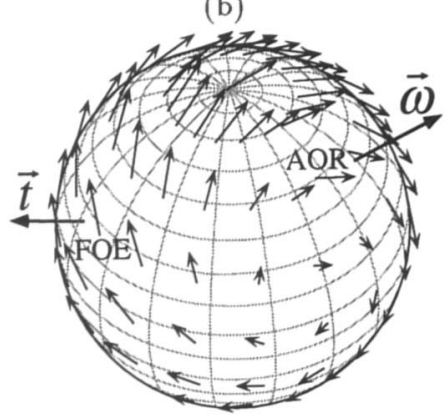

(c)

Figure 2. Example of (a) a rotational, (b) a translational, and (c) a general motion field on a sphere. FOE and AOR denote the intersection of $\mathbf{t}$ and $\boldsymbol{\omega}$ with the sphere, respectively.

translation. If all we have is the direction of the flow we can project $\dot{\mathbf{r}}$ onto any unit vector $\mathbf{n}_{i}$ on the image and obtain an inequality constraint:

$\dot{\mathbf{r}} \cdot \mathbf{n}_{i}=\left(\frac{1}{|\mathbf{R}|}((\mathbf{t} \cdot \mathbf{r}) \mathbf{r}-\mathbf{t})-\boldsymbol{\omega} \times \mathbf{r}\right) \cdot \mathbf{n}_{i}>0 \quad$ or $<0$

From this inequality we certainly cannot recover the magnitude of translation, since the optical flow already does not allow us to compute it.

In addition we are also restricted in the computation of the rotational parameters. If we multiply $\boldsymbol{\omega}$ by a positive constant, leave $\mathbf{t}$ fixed, but multiply $1 /|\mathbf{R}|$ by the same positive constant, the sign of the flow is not affected. Thus from the direction of the flow we can at most compute the axis of rotation and, as discussed before, the axis of translation. Hereafter, for the sake of brevity, we will refer to the motion field also as the flow field or simply flow, and to the direction of the motion field as the directional flow field or simply directional flow.

\section{Relationship between the Orientation of the Flow and the Depth-Positivity Constraint}

If we have the flow $\dot{\mathbf{r}}$, we know the value of the projection of $\dot{\mathbf{r}}$ on any direction and we get all the possible information by choosing two directions $\mathbf{n}_{1}$ and $\mathbf{n}_{2}$ (usually orthogonal). Thus we have

$$
\begin{array}{r}
\dot{\mathbf{r}} \cdot \mathbf{n}_{i}=\frac{1}{|\mathbf{R}|}((\mathbf{t} \cdot \mathbf{r}) \mathbf{r}-\mathbf{t}) \cdot \mathbf{n}_{i}-(\boldsymbol{\omega} \times \mathbf{r}) \cdot \mathbf{n}_{i} \\
\text { for } i=1,2
\end{array}
$$

We can solve Eq. (1) for the depth,

$$
\frac{\dot{\mathbf{r}} \cdot \mathbf{n}_{i}+(\boldsymbol{\omega} \times \mathbf{r}) \cdot \mathbf{n}_{i}}{((\mathbf{t} \cdot \mathbf{r}) \mathbf{r}-\mathbf{t}) \cdot \mathbf{n}_{i}}=\frac{1}{|\mathbf{R}|} \quad \text { for } i=1,2
$$

Knowing the value in both directions $\mathbf{n}_{1}$ and $\mathbf{n}_{2}$ we know that the inverse depth has to be the same, and also has to be positive; thus

$$
\frac{\dot{\mathbf{r}} \cdot \mathbf{n}_{1}+(\boldsymbol{\omega} \times \mathbf{r}) \cdot \mathbf{n}_{1}}{((\mathbf{t} \cdot \mathbf{r}) \mathbf{r}-\mathbf{t}) \cdot \mathbf{n}_{1}}=\frac{\dot{\mathbf{r}} \cdot \mathbf{n}_{2}+(\boldsymbol{\omega} \times \mathbf{r}) \cdot \mathbf{n}_{2}}{((\mathbf{t} \cdot \mathbf{r}) \mathbf{r}-\mathbf{t}) \cdot \mathbf{n}_{2}}>0
$$

If on the other hand we do not use the value of the flow but only its direction and thus the sign of the projection of the flow on $\mathbf{n}_{i}$, then the only constraint that can be utilized is the inequality, which comes from the fact that the depth is positive. Using only the orientation of the flow we obtain for every direction $\mathbf{n}_{i}$

$$
\frac{\dot{\mathbf{r}} \cdot \mathbf{n}_{i}+(\boldsymbol{\omega} \times \mathbf{r}) \cdot \mathbf{n}_{i}}{((\mathbf{t} \cdot \mathbf{r}) \mathbf{r}-\mathbf{t}) \cdot \mathbf{n}_{i}}>0
$$

This inequality provides inequality constraints on the rotational and translational components, which are independent of the scene: If we consider the sign of the translational component $((\mathbf{t} \cdot \mathbf{r}) \mathbf{r}-\mathbf{t}) \cdot \mathbf{n}_{i}$ and the sign of the rotational component $(\boldsymbol{\omega} \times \mathbf{r}) \cdot \mathbf{n}_{i}$ and assume that each of them is either positive or negative, there are $2 \times 2=4$ combinations of signs. But once we know the sign of the flow $\dot{\mathbf{r}} \cdot \mathbf{n}_{i}$, one of these four combinations is no longer possible. This observation has been 
used in the development of global constraints for 3D motion estimation. Choosing directions $\mathbf{n}_{i}$ in particular ways the signs of $\left(\dot{\mathbf{r}} \cdot \mathbf{n}_{i}\right)$ form global patterns of positive and negative areas on the image (Fermüller, 1995; Fermüller and Aloimonos, 1995, 1996). These patterns, whose location and form encodes information about 3D motion, were successfully used in the recovery of egomotion. In this paper, by pursuing a theoretical investigation of the amount of information present in directional flow fields, we demonstrate the power of the qualitative image measurements already used empirically, and justify their utilization in global constraints for three-dimensional dynamic vision problems.

The organization of this paper is as follows: In Section 3 we develop the preliminaries-constraints that will be used in the uniqueness analysis. Given two rigid motions, we study what the constraints are on the surfaces in view for the two motion fields to have the same direction at every point. From these constraints, we investigate for which points of the image one of the surfaces must have negative depth. The locations where negative depth occurs are described implicitly in the form of constraints on the signs of functions depending on the image coordinates and the two three-dimensional motions. The existence of image points whose associated depth is negative ensures that the two rigid motions cannot produce motion fields with the same direction. In Section 4, which contains the main uniqueness proof, we study conditions under which two rigid flow fields could have the same direction at every point on a half sphere (i.e., conditions under which there do not exist points of negative depth), and we visualize the locations of negative depth on the sphere. Section 5 is devoted to the treatment of special cases. Section 6 studies whether more than two rigid motions could produce the same directional flow field. As a byproduct of the analysis, in Section 7 we investigate the ambiguity of rigid motion for full flow assuming that depth has to be positive, and show that any two different motions can be distinguished on a hemispherical image from full flow. Section 8 summarizes the results. The Appendices describe and prove a number of geometric properties used in the main part of the paper.

\section{Critical Surface Constraints}

Let us assume that two different rigid motions yield the same directional flow at every point in the image.
Let $\mathbf{t}_{1}$ and $\boldsymbol{\omega}_{1}$ be translational and rotational velocities of the first motion, and let $\mathbf{t}_{2}$ and $\boldsymbol{\omega}_{2}$ be translational and rotational velocities of the second motion. To simplify the explanations, we assume $\mathbf{t}_{1} \times \mathbf{t}_{2} \neq 0$ and $\boldsymbol{\omega}_{1} \times \boldsymbol{\omega}_{2} \neq 0$. The special cases are dealt with in Section 5 .

Since from the direction of flow we can only recover the directions of the translation and rotation axes, we assume all four vectors $\mathbf{t}_{1}, \mathbf{t}_{2}, \boldsymbol{\omega}_{1}$ and $\boldsymbol{\omega}_{2}$ to be of unit length. Let $Z_{1}(\mathbf{r})$ and $Z_{2}(\mathbf{r})$ be the functions, mapping points $\mathbf{r}$ on the image onto the real numbers, that represent the depths of the surfaces in view corresponding to the two motions. In the future we will refer to $Z_{1}$ and $Z_{2}$ as the two depth maps. We assume that the two depths are positive, and allow $Z_{1}$ or $Z_{2}$ to be infinitely large. Thus we assume $1 / Z_{1} \geq 0$ and $1 / Z_{2} \geq 0$.

In this section we investigate the constraints that must be satisfied by $Z_{1}$ and $Z_{2}$ in order for the two flow fields to have the same direction.

\subsection{Notation}

We start by defining some notation:

$$
\begin{aligned}
f_{\omega}(\mathbf{r}) & =\left[\boldsymbol{\omega}_{1} \boldsymbol{\omega}_{2} \mathbf{r}\right] \\
f_{t}(\mathbf{r}) & =\left[\mathbf{t}_{1} \mathbf{t}_{2} \mathbf{r}\right] \\
g_{i j}(\mathbf{r}) & =\left(\boldsymbol{\omega}_{i} \times \mathbf{r}\right) \cdot\left(\mathbf{t}_{j} \times \mathbf{r}\right) \quad \text { for } i, j=1,2
\end{aligned}
$$

where $[\mathbf{a b c}]=(\mathbf{a} \times \mathbf{b}) \cdot \mathbf{c}$ denotes the triple product of vectors $\mathbf{a}, \mathbf{b}$ and $\mathbf{c}$.

These functions have a simple geometric meaning. Function $f_{\omega}(\mathbf{r})$ is zero for points $\mathbf{r}$ lying on a geodesic passing through $\boldsymbol{\omega}_{1}$ and $\boldsymbol{\omega}_{2}$. The geodesic is the locus of points $\mathbf{r}$ where $v_{\text {rot }_{1}}(\mathbf{r})$, the rotational component of the first motion, is parallel to $v_{\text {rot }_{2}}(\mathbf{r})$, the rotational component of the second motion. Similarly, the geodesic passing through $\mathbf{t}_{1}$ and $\mathbf{t}_{2}$ is the locus of points where $f_{t}(\mathbf{r})=0$ and $v_{\mathrm{tr}_{1}}(\mathbf{r})$ is parallel to $v_{\mathrm{tr}_{2}}(\mathbf{r})$.

Equation $g_{i j}(\mathbf{r})=0$ defines a second-order contour consisting of two closed curves on the sphere, the socalled zero motion contour of motion $\left(\mathbf{t}_{j}, \boldsymbol{\omega}_{i}\right)$. It is the locus of points where $v_{\mathrm{rot}_{i}}(\mathbf{r})$ is parallel to $v_{\mathrm{tr}_{j}}(\mathbf{r})$, and therefore the locus of points where the flow due to the motion $\left(\mathbf{t}_{j}, \boldsymbol{\omega}_{i}\right)$ could be zero (see Appendix A). Throughout the paper the functions $f_{i}(\mathbf{r}), g_{i j}(\mathbf{r})$ and the curves defined by their zero crossings will play very important roles.

To simplify the notation we will usually drop $\mathbf{r}$ and write only $f_{i}$ and $g_{i j}$ where the index $i$ in $f_{i}$ can take values $t$ and $\omega$. There is a simple relationship between 
$f_{i}$ and $g_{i j}$. Let

$$
\begin{aligned}
\mathbf{u}_{1} & =\left(\mathbf{r} \times\left(\mathbf{t}_{1} \times \mathbf{r}\right)\right) \times\left(\boldsymbol{\omega}_{1} \times \mathbf{r}\right) \\
& =-\left[\boldsymbol{\omega}_{1} \mathbf{r}\left(\mathbf{t}_{1} \times \mathbf{r}\right)\right] \mathbf{r}=-g_{11} \mathbf{r} \\
\mathbf{u}_{2} & =\left(\mathbf{r} \times\left(\mathbf{t}_{2} \times \mathbf{r}\right)\right) \times\left(\boldsymbol{\omega}_{2} \times \mathbf{r}\right)=-g_{22} \mathbf{r}
\end{aligned}
$$

Since we assume $(\mathbf{r} \cdot \mathbf{r})=1$, we obtain

$$
\mathbf{u}_{1} \cdot \mathbf{u}_{2}=g_{11} g_{22}
$$

Let

$$
\mathbf{u}_{3}=\left(\mathbf{t}_{2} \times \mathbf{r}\right) \times\left(\mathbf{r} \times\left(\boldsymbol{\omega}_{2} \times \mathbf{r}\right)\right)=-\mathbf{u}_{2}
$$

Then

$$
\mathbf{u}_{1} \cdot \mathbf{u}_{2}=-\mathbf{u}_{1} \cdot \mathbf{u}_{3}=f_{t} f_{\omega}+g_{12} g_{21}
$$

From Eqs. (3) and (4) we obtain

$$
g_{11} g_{22}=f_{t} f_{\omega}+g_{12} g_{21}
$$

\subsection{Conditions for Ambiguity}

Assume that motion $\left(\mathbf{t}_{1}, \boldsymbol{\omega}_{1}\right)$ with depth map $Z_{1}$ and motion $\left(\mathbf{t}_{2}, \boldsymbol{\omega}_{2}\right)$ with depth map $Z_{2}$ give rise to flow fields with the same direction at any point; then there exists $\mu>0$ such that

$$
\begin{aligned}
- & \frac{1}{Z_{1}}\left(\mathbf{r} \times\left(\mathbf{t}_{1} \times \mathbf{r}\right)\right)-\omega_{1} \times \mathbf{r} \\
\quad & \mu\left(-\frac{1}{Z_{2}}\left(\mathbf{r} \times\left(\mathbf{t}_{2} \times \mathbf{r}\right)\right)-\omega_{2} \times \mathbf{r}\right)
\end{aligned}
$$

By projecting the vector Eq. (6) on directions $\mathbf{t}_{2} \times \mathbf{r}$ and $\mathbf{r} \times\left(\boldsymbol{\omega}_{2} \times \mathbf{r}\right)$ we obtain two scalar equations

$$
\begin{aligned}
& \frac{1}{Z_{1}}\left[\mathbf{t}_{1} \mathbf{t}_{2} \mathbf{r}\right]+\left(\boldsymbol{\omega}_{1} \times \mathbf{r}\right) \cdot\left(\mathbf{t}_{2} \times \mathbf{r}\right) \\
& \quad=\mu\left(\boldsymbol{\omega}_{2} \times \mathbf{r}\right) \cdot\left(\mathbf{t}_{2} \times \mathbf{r}\right) \\
& \frac{1}{Z_{1}}\left(\boldsymbol{\omega}_{2} \times \mathbf{r}\right) \cdot\left(\mathbf{t}_{1} \times \mathbf{r}\right)-\left[\boldsymbol{\omega}_{1} \boldsymbol{\omega}_{2} \mathbf{r}\right] \\
& \quad=\mu \frac{1}{Z_{2}}\left(\boldsymbol{\omega}_{2} \times \mathbf{r}\right) \cdot\left(\mathbf{t}_{2} \times \mathbf{r}\right)
\end{aligned}
$$

Since $\mu$ is positive, from (7) and (8) we get constraints on $\frac{1}{Z_{1}}$ :

$$
\operatorname{sgn}\left(\frac{1}{Z_{1}} f_{t}+g_{12}\right)=\operatorname{sgn}\left(g_{22}\right)
$$

$$
\operatorname{sgn}\left(\frac{1}{Z_{1}} g_{21}-f_{\omega}\right)=\operatorname{sgn}\left(\frac{1}{Z_{2}} g_{22}\right)
$$

where $\operatorname{sgn}(\cdot)$ denotes the sign function.

Let us define $s_{1}=-g_{12} / f_{t}$ and $s_{1}^{\prime}=f_{\omega} / g_{21}$. At any point, $f_{i}$ and $g_{i j}$ are constant, so Eqs. (9) and (10) provide simple constraints on $1 / Z_{1}$. Equation (9) provides the following relationship between $1 / Z_{1}$ and $s_{1}$ : if $\operatorname{sgn}\left(g_{22}\right) \operatorname{sgn}\left(f_{t}\right)><0$ then $1 / Z_{1}><s_{1}$; if $\operatorname{sgn}\left(g_{22}\right)=0$ then $1 / Z_{1}=s_{1}$, and if $\operatorname{sgn}\left(f_{t}\right)=0$ then $1 / Z_{1}$ is not constrained by (9). We thus call it the $s_{1}$-constraint. Similarly, we refer to Eq. (10) as the $s_{1}^{\prime}$-constraint since it provides the following relationship between $1 / Z_{1}$ and $s_{1}^{\prime}$ : if $\operatorname{sgn}\left(g_{22}\right) \operatorname{sgn}\left(f_{21}\right)><0$ then $1 / Z_{1}><s_{1}^{\prime}$; if $\operatorname{sgn}\left(g_{22}\right)=0$ then $1 / Z_{1}=s_{1}^{\prime}$, and if $\operatorname{sgn}\left(g_{21}\right)=0$ then $1 / Z_{1}$ is not constrained by (10).

Let us now interpret these constraints: $1 / Z_{2}$ is always non-negative; thus, if the two motions $\left(\mathbf{t}_{1}, \boldsymbol{\omega}_{1}\right)$, $\left(\mathbf{t}_{2}, \boldsymbol{\omega}_{2}\right)$ with their corresponding depth maps $Z_{1}$ and $Z_{2}$ produce flow with the same direction, the depth $Z_{1}$ must satisfy either

$$
\frac{1}{Z_{1}} f_{t}+g_{12}>0 \quad \text { and } \quad \frac{1}{Z_{1}} g_{21}-f_{\omega} \geq 0
$$

or

$$
\frac{1}{Z_{1}} f_{t}+g_{12}<0 \quad \text { and } \quad \frac{1}{Z_{1}} g_{21}-f_{\omega} \leq 0
$$

Thus $Z_{1}$ has a relationship to the surfaces

and

$$
Z(\mathbf{r})=\frac{1}{s_{1}(\mathbf{r})}
$$

$$
Z(\mathbf{r})=\frac{1}{s_{1}^{\prime}(\mathbf{r})}
$$

Equations (11) and (12) provide hybrid definitions of scene surfaces. To express the surfaces in scene coordinates $\mathbf{R}$, we substitute in the above equations $Z(\mathbf{r}) \mathbf{r}=\mathbf{R}$. Dividing $(11)$ by $Z(\mathbf{r})$ and replacing $Z(\mathbf{r})^{2}$ by $\mathbf{R}^{2}$ in (12) we obtain

$$
\left(\mathbf{t}_{1} \times \mathbf{t}_{2}\right) \cdot \mathbf{R}+\left(\boldsymbol{\omega}_{1} \times \mathbf{R}\right) \cdot\left(\mathbf{t}_{2} \times \mathbf{R}\right)=0
$$

and

$$
\left(\boldsymbol{\omega}_{2} \times \mathbf{R}\right) \cdot\left(\mathbf{t}_{1} \times \mathbf{R}\right)-\left(\left(\boldsymbol{\omega}_{1} \times \boldsymbol{\omega}_{2}\right) \cdot \mathbf{R}\right) \mathbf{R}^{2}=0
$$

Thus we see that $Z_{1}$ is constrained by a secondorder surface through (13) and by a third-order surface through (14). At some points it has to be inside the first 


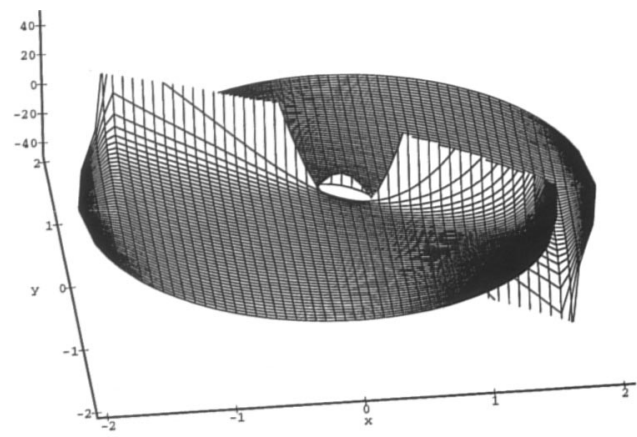

(a)

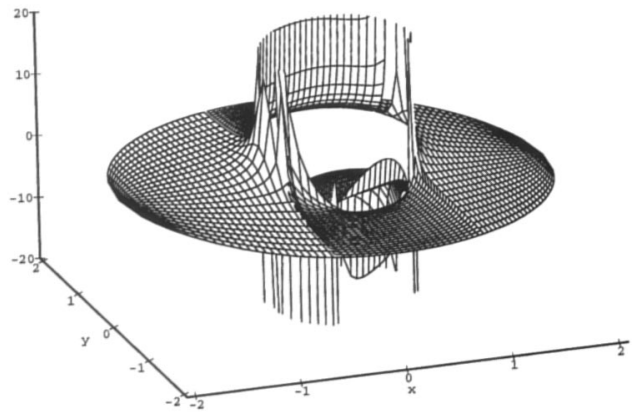

(b)

Figure 3. Two rigid motions $\left(\mathbf{t}_{1}, \boldsymbol{\omega}_{1}\right),\left(\mathbf{t}_{2}, \boldsymbol{\omega}_{2}\right)$ constrain the possible depth $Z_{1}$ of the first surface by (a) a second- and (b) a third-order surface. The particular surfaces shown in the coordinate system of the imaging sphere, projected stereographically, correspond to the motion configuration of Fig. 5.

surface and at some points it has to be outside the first surface. In addition, at some points it has to be inside the second surface and at some points it has to be outside the second surface. Figure 3 provides a pictorial description of the two surfaces constraining $Z_{1}$.

We can repeat the above derivation for the depth map $Z_{2}$. Projecting Eq. (6) onto vectors $\mathbf{t}_{1} \times \mathbf{r}$ and $\mathbf{r} \times\left(\boldsymbol{\omega}_{1} \times \mathbf{r}\right)$, we obtain constraints on $1 / Z_{2}$ :

$$
\begin{array}{r}
\operatorname{sgn}\left(g_{11}\right)=\operatorname{sgn}\left(-\frac{1}{Z_{2}} f_{t}+g_{21}\right) \\
\operatorname{sgn}\left(\frac{1}{Z_{1}} g_{11}\right)=\operatorname{sgn}\left(\frac{1}{Z_{2}} g_{12}+f_{\omega}\right)
\end{array}
$$

We define $s_{2}=g_{21} / f_{t}, s_{2}^{\prime}=-f_{\omega} / g_{12}$. Equations (15) and (16) provide constraints on $1 / Z_{2}$, and we call them the $s_{2}$-constraint and the $s_{2}^{\prime}$-constraint. From these equations we obtain a further second- and thirdorder surface pair, which constrain the depth map $Z_{2}$.

\subsection{Interpretation of Surface Constraints}

We next describe the $s_{1}$ - and $s_{1}^{\prime}$-constraints in detail. For convenience, we express these constraints for $1 / Z_{1}$.

If $f_{t}=0$, then the $s_{1}$-constraint is $\operatorname{sgn}\left(g_{12}\right)=$ $\operatorname{sgn}\left(g_{22}\right)$, i.e., it does not depend on $Z_{1}$. Thus it is either satisfied by any $Z_{1}$, or it cannot be satisfied by any $Z_{1}$.

If $f_{t} \neq 0$, then we get the $s_{1}$-constraint $\operatorname{sgn}\left(\frac{1}{Z_{1}}-s_{1}\right)$ $=\operatorname{sgn}\left(g_{22}\right) \operatorname{sgn}\left(f_{t}\right)$. So the $s_{1}$-constraint is

- $1 / Z_{1}>s_{1}$, if $\operatorname{sgn}\left(g_{22}\right) \operatorname{sgn}\left(f_{t}\right)>0$

- $1 / Z_{1}=s_{1}$, if $\operatorname{sgn}\left(g_{22}\right)=0$

- $1 / Z_{1}<s_{1}$, if $\operatorname{sgn}\left(g_{22}\right) \operatorname{sgn}\left(f_{t}\right)<0$

If $g_{21}=0$, then the $s_{1}^{\prime}$-constraint does not depend on $Z_{1}$.

If $g_{21} \neq 0$, we get $\operatorname{sgn}\left(\frac{1}{Z_{1}}-s_{1}^{\prime}\right)=\operatorname{sgn}\left(\frac{1}{Z_{2}} g_{22}\right)$ $\operatorname{sgn}\left(g_{21}\right)$. Since we assume $1 / Z_{2} \geq 0, \operatorname{sgn}\left(\frac{1}{Z_{2}} g_{22}\right)$ is either 0 , or $\operatorname{sgn}\left(g_{22}\right)$. So the $s_{1}^{\prime}$-constraint is

- $1 / Z_{1} \geq s_{1}^{\prime}$, if $\operatorname{sgn}\left(g_{22}\right) \operatorname{sgn}\left(g_{21}\right)>0$

- $1 / Z_{1}=s_{1}^{\prime}$, if $\operatorname{sgn}\left(g_{22}\right)=0$

- $1 / Z_{1} \leq s_{1}^{\prime}$, if $\operatorname{sgn}\left(g_{22}\right) \operatorname{sgn}\left(g_{21}\right)<0$

At each point we have the additional constraint $1 / Z_{1} \geq 0$. If all three constraints can be satisfied simultaneously at a point in the image, then there is an interval (bounded or unbounded) of values of $Z_{1}$ satisfying them. If the constraints cannot be satisfied, this means that the two flows at this point cannot have the same direction and we say that we have a contradictory point.

In Table 1 we summarize the three constraints on $1 / Z_{1}$. The table analyzes the general case, at a point where $f_{i} \neq 0$ and $g_{i j} \neq 0$.

If some of $f_{i}, g_{i j}$ are zero at a point, we may obtain constraints that do not depend on $Z_{1}$, or equality constraints.

The classification of a point according to the $s_{1^{-}}$ constraint and $s_{1}^{\prime}$-constraint depends on the signs of $f_{t}, g_{21}$, and $g_{22}$. The existence of a solution interval at a point also depends on the signs of $f_{\omega}$ and $g_{12}$ at that point and also on the relative values of $s_{1}$ and $s_{1}^{\prime}$, i.e., on the sign of $s_{1}-s_{1}^{\prime}$.

Functions $f_{i}(\mathbf{r})$ and $g_{i j}(\mathbf{r})$ are polynomial functions of $\mathbf{r}$. To find out where they change sign, it is enough to find points where they are zero. The sign of $s_{1}-s_{1}^{\prime}$ is more complicated, since $s_{1}(\mathbf{r})$ and $s_{1}^{\prime}(\mathbf{r})$ do not have to be continuous. However, their discontinuities occur at points where $f_{t}(\mathbf{r})=0$ or $g_{21}(\mathbf{r})=0$. Thus $\operatorname{sgn}\left(s_{1}-s_{1}^{\prime}\right)$ 
Table 1. Classification of ambiguous points.

\begin{tabular}{lcccc}
\hline Type & Positivity constraint & $s_{1}$-constraint & $s_{1}^{\prime}$-constraint & Solution exists if \\
\hline I & $\frac{1}{Z_{1}} \geq 0$ & $\frac{1}{Z_{1}}>s_{1}$ & $\frac{1}{Z_{1}} \geq s_{1}^{\prime}$ & Always \\
II & $\frac{1}{Z_{1}} \geq 0$ & $\frac{1}{Z_{1}}>s_{1}$ & $\frac{1}{Z_{1}} \leq s_{1}^{\prime}$ & $s_{1}^{\prime} \geq 0$ and $s_{1}<s_{1}^{\prime}$ \\
III & $\frac{1}{Z_{1}} \geq 0$ & $\frac{1}{Z_{1}}<s_{1}$ & $\frac{1}{Z_{1}} \geq s_{1}^{\prime}$ & $s_{1}>0$ and $s_{1}^{\prime}<s_{1}$ \\
IV & $\frac{1}{Z_{1}} \geq 0$ & $\frac{1}{Z_{1}}<s_{1}$ & $\frac{1}{Z_{1}} \leq s_{1}^{\prime}$ & $s_{1}>0$ and $s_{1}^{\prime} \geq 0$ \\
\hline
\end{tabular}

can change at those points and at points where $s_{1}-s_{1}^{\prime}$ $=0$. Using (5), we can write

$$
\begin{aligned}
s_{1}-s_{1}^{\prime} & =-\frac{g_{12}}{f_{t}}-\frac{f_{\omega}}{g_{21}} \\
& =-\frac{1}{f_{t} g_{21}}\left(f_{t} f_{\omega}+g_{12} g_{21}\right)=-\frac{g_{11} g_{22}}{f_{t} g_{21}}
\end{aligned}
$$

So we see that $\operatorname{sgn}\left(s_{1}-s_{1}^{\prime}\right)$ can change only at points where at least one of $f_{i}, g_{i j}$ is zero. At points where $g_{22}=0$ we have the $s_{1}$-constraint $1 / Z_{1}=s_{1}$ and the $s_{1}^{\prime}$-constraint $1 / Z_{1}=s_{1}^{\prime}$. From Eq. (17) we obtain $s_{1}=s_{1}^{\prime}$; thus at these points the depth $Z_{1}$ is uniquely defined.

Up to this point we have been discussing only the constraints for $Z_{1}$. Similarly, from (15) and (16) we have at any point the $s_{2}$-constraint $\left(1 / Z_{2}>s_{2}\right.$, $1 / Z_{2}=s_{2}$, or $\left.1 / Z_{2}<s_{2}\right)$, and the $s_{2}^{\prime}$-constraint $\left(1 / Z_{2} \geq s_{2}^{\prime}, 1 / Z_{2}=s_{2}^{\prime}\right.$, or $\left.1 / Z_{2} \leq s_{2}^{\prime}\right)$. Again, the type of solution for $Z_{2}$ can change only at points where at least one of $f_{i}, g_{i j}$ is zero.

Now we can summarize the results. The curves $f_{i}(\mathbf{r})=0$ and $g_{i j}(\mathbf{r})=0$ separate the sphere into a number of areas (see Fig. 5 for an example). Each of the areas is either contradictory (i.e., containing only contradictory points), or ambiguous (i.e., containing points where the two motion vectors can have the same direction). Two different rigid motions can produce ambiguous directions of flow if the image contains only points from ambiguous areas. There are also two scene surfaces constraining depth $Z_{1}$ and two surfaces constraining depth $Z_{2}$. If the depths do not satisfy the constraints, the two flows are not ambiguous.

\subsection{Contradictory Points}

An image point is contradictory if the signs of $f_{i}$ and $g_{i j}$ satisfy certain conditions. Since we are interested in contradictory areas, we investigate only the general case, i.e., assume $f_{i} \neq 0$ and $g_{i j} \neq 0$ and use the resulting constraints in Section 4. If $\mathbf{t}_{1} \times \mathbf{t}_{2}=0$ or $\boldsymbol{\omega}_{1} \times \boldsymbol{\omega}_{2}=0$, this assumption is violated everywhere in the image. Such cases are treated separately in Section 5.

There are two simple conditions yielding contradiction for $Z_{1}$, one for the $s_{1}$-constraint and one for the $s_{1}^{\prime}$-constraint. There is no solution for $Z_{1}$ if $1 / Z_{1}<s_{1}$ and $s_{1} \leq 0$. This happens under the following condition $C_{1}$ :

$$
\operatorname{sgn}\left(f_{t}\right)=\operatorname{sgn}\left(g_{12}\right)=-\operatorname{sgn}\left(g_{22}\right)
$$

which is derived from Eq. (9). Similarly, from (10) we get a contradiction if $1 / Z_{1} \leq s_{1}^{\prime}$ and $s_{1}^{\prime}<0$, i.e., under condition $C_{2}$ :

$$
\operatorname{sgn}\left(f_{\omega}\right)=-\operatorname{sgn}\left(g_{21}\right)=\operatorname{sgn}\left(g_{22}\right)
$$

We get similar conditions for $Z_{2}$. There is no solution for $Z_{2}$, if $1 / Z_{2}<s_{2}$ and $s_{2} \leq 0$, or if $1 / Z_{2} \leq s_{2}^{\prime}$ and $s_{2}^{\prime}<0$. This happens under conditions $C_{3}$ and $C_{4}$ :

$$
\operatorname{sgn}\left(f_{t}\right)=-\operatorname{sgn}\left(g_{21}\right)=\operatorname{sgn}\left(g_{11}\right)
$$

and

$$
\operatorname{sgn}\left(f_{\omega}\right)=\operatorname{sgn}\left(g_{12}\right)=-\operatorname{sgn}\left(g_{11}\right)
$$

We call these four constraints $\left(C_{1}-C_{4}\right)$ Contradictory Point conditions, or $\mathrm{CP}$-conditions for short. Next we show that a point (where $f_{i} \neq 0$ and $g_{i j} \neq 0$ ) is contradictory if and only if at least one of the four conditions is satisfied.

Let us assume that conditions (18) and (19) are not satisfied at some point, but we have a contradiction for $Z_{1}$. This is possible if either $1 / Z_{1} \leq s_{1}^{\prime}<s_{1}<1 / Z_{1}$, or if $1 / Z_{1}<s_{1}<s_{1}^{\prime} \leq 1 / Z_{1}$.

Both conditions occur when $\operatorname{sgn}\left(g_{22} g_{21}\right)=-\sigma$, $\operatorname{sgn}\left(-g_{11} g_{22} f_{t} g_{21}\right)=\sigma$, and $\operatorname{sgn}\left(g_{22} f_{t}\right)=\sigma$, with $\sigma=1$ in the first case and $\sigma=-1$ in the second case. Multiplying the equations by $-\operatorname{sgn}\left(g_{22} f_{t} g_{21}\right)$, 
we obtain $\operatorname{sgn}\left(f_{t}\right)=\operatorname{sgn}\left(g_{11}\right)=-\operatorname{sgn}\left(g_{21}\right)$, i.e., condition (20).

Thus if there is no solution for $Z_{1}$, at least one of conditions (18), (19), and (20) must hold. Similarly if there is no solution for $Z_{2}$, at least one of conditions (18), (20), and (21) must hold. Therefore the CP-conditions provide a complete description of the contradictory areas in the general case.

By examination of all the possibilities, we can show that at any point, either none of the CP-conditions holds (and the point is ambiguous), or exactly two of the conditions hold (and the point is contradictory).

\subsection{Antipodal Pairs of Points}

Let us now consider a point $\mathbf{r}$ and its antipodal point $-\mathbf{r}$ such that $f_{i}(\mathbf{r}) \neq 0$ and $g_{i j}(\mathbf{r}) \neq 0$.

Clearly, $f_{t}(-\mathbf{r})=-f_{t}(\mathbf{r}), f_{\omega}(-\mathbf{r})=-f_{\omega}(\mathbf{r})$, but $g_{i j}(-\mathbf{r})=g_{i j}(\mathbf{r})$. If $\operatorname{sgn}\left(g_{12}(\mathbf{r})\right) \neq \operatorname{sgn}\left(g_{22}(\mathbf{r})\right)$, then condition (18) holds either at $\mathbf{r}$, or at $-\mathbf{r}$. We get similar results for the remaining three $\mathrm{CP}$-conditions.

Thus both point $\mathbf{r}$ and point $-\mathbf{r}$ are ambiguous only if

$$
\begin{aligned}
\operatorname{sgn}\left(g_{11}(\mathbf{r})\right) & =\operatorname{sgn}\left(g_{12}(\mathbf{r})\right) \\
& =\operatorname{sgn}\left(g_{21}(\mathbf{r})\right)=\operatorname{sgn}\left(g_{22}(\mathbf{r})\right)
\end{aligned}
$$

\section{The Geometry of the Depth-Positivity Constraint}

In the last section we found that if the CP-conditions hold at a point on the imaging surface, then one of the depth values has to be negative and thus the point is contradictory. In this section we investigate these constraints further; in particular, we would like to know under what conditions two rigid motions cannot be distinguished if our imaging surface is a half sphere or an image plane, and we are interested in studying and visualizing the locations of areas where the $\mathrm{CP}$-conditions are met.

Considering as imaging surface the whole sphere, two different rigid motions cannot produce flow of the same direction everywhere. As shown in Section 3.5, two antipodal points $\mathbf{r}$ and $-\mathbf{r}$ are ambiguous only if (22) holds. If the two motions are different, there always exist areas on the sphere where this condition is not satisfied. Since for any point the image also contains its antipodal point, clearly some areas in the image are contradictory.
We are now ready, using the machinery already developed, to study uniqueness properties. In the sequel we assume that the image is a half of the sphere. Let the image hemisphere be bounded by equator $q$ and let $\mathbf{n}_{0}$ be a unit vector normal to the plane of $q$.

\subsection{Half Sphere Image: The General Case}

Let us assume that

$$
\left(\boldsymbol{\omega}_{1} \times \boldsymbol{\omega}_{2}\right) \cdot\left(\mathbf{t}_{1} \times \mathbf{t}_{2}\right) \neq 0
$$

We show that under this condition the two rigid motions cannot produce motion fields with the same direction everywhere in the image.

Let us consider the projections of $\boldsymbol{\omega}_{1}$ and $\boldsymbol{\omega}_{2}$ on the geodesic $n$ connecting $\mathbf{t}_{1}$ and $\mathbf{t}_{2}$. Projection onto the geodesic is well defined for all points $\mathbf{r}$ such that $\mathbf{r} \times\left(\mathbf{t}_{1} \times \mathbf{t}_{2}\right) \neq 0$. Since we assume (23), the projections of both $\boldsymbol{\omega}_{1}$ and $\boldsymbol{\omega}_{2}$ are well defined. The proof is given in parts $\mathrm{A}$ and $\mathrm{B}$.

A: Let us first assume that one of $\boldsymbol{\omega}_{1}, \boldsymbol{\omega}_{2}$ does not lie on geodesic $n$. Without loss of generality, let it be $\boldsymbol{\omega}_{1}$, i.e., let $\left[\mathbf{t}_{1} \mathbf{t}_{2} \boldsymbol{\omega}_{1}\right] \neq 0$.

The projection of $\boldsymbol{\omega}_{1}$ onto $n$ is

$$
\mathbf{r}_{1}= \pm\left(\mathbf{t}_{1} \times \mathbf{t}_{2}\right) \times\left(\boldsymbol{\omega}_{1} \times\left(\mathbf{t}_{1} \times \mathbf{t}_{2}\right)\right)
$$

where the sign is chosen so that $\mathbf{r}_{1}$ is in the image. Then

$$
\begin{aligned}
f_{\omega}\left(\mathbf{r}_{1}\right) & = \pm\left(\left(\boldsymbol{\omega}_{1} \times \boldsymbol{\omega}_{2}\right) \times\left(\mathbf{t}_{1} \times \mathbf{t}_{2}\right)\right) \cdot\left(\boldsymbol{\omega}_{1} \times\left(\mathbf{t}_{1} \times \mathbf{t}_{2}\right)\right) \\
& =\mp\left[\mathbf{t}_{1} \mathbf{t}_{2} \boldsymbol{\omega}_{1}\right]\left(\left(\boldsymbol{\omega}_{1} \times \boldsymbol{\omega}_{2}\right) \cdot\left(\mathbf{t}_{1} \times \mathbf{t}_{2}\right)\right) \neq 0
\end{aligned}
$$

and $g_{11}\left(\mathbf{r}_{1}\right)=g_{12}\left(\mathbf{r}_{1}\right)=0$. So the $s_{2}^{\prime}$-constraint is $\operatorname{sgn}\left(1 / Z_{1} 0\right)=\operatorname{sgn}\left(\frac{1}{Z_{2}} 0+f_{\omega}\right)$ or $f_{\omega}=0$. Clearly, this constraint cannot be satisfied, so $\mathbf{r}_{1}$ is a contradictory point.

We can also show that at least one of the areas around point $\mathbf{r}_{1}$ is contradictory. Point $\mathbf{r}_{1}$ lies on zero motion contours $g_{11}(\mathbf{r})=0$ and $g_{12}(\mathbf{r})=0$. Since $\mathbf{t}_{1} \times \mathbf{t}_{2} \neq 0$, contours $g_{11}$ and $g_{12}$ must cross at a point $\mathbf{r}_{1}$. (This can be shown using Appendix $\mathrm{C}$, which describes under what circumstances zero motion contours are tangent.)

Thus we obtain four areas in the neighborhood of $\mathbf{r}_{1}$ with all four possible sign combinations of $g_{11}$ and $g_{12}$. If we look at points close enough to $\mathbf{r}_{1}$ (so that $f_{\omega}$ does not change sign), then condition (21) is satisfied in one of the areas, and that area is contradictory. For an illustration see Fig. 4. 


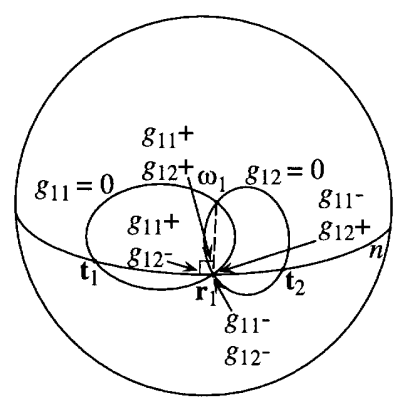

Figure 4. Possible sign combinations of $g_{11}$ and $g_{12}$ in the neighborhood of $\mathbf{r}_{1}$.

B: Now we need to consider the situation where both $\boldsymbol{\omega}_{1}$ and $\boldsymbol{\omega}_{2}$ lie on geodesic $n$, i.e., $\left[\mathbf{t}_{1} \mathbf{t}_{2} \boldsymbol{\omega}_{1}\right]=$ $\left[\mathbf{t}_{1} \mathbf{t}_{2} \boldsymbol{\omega}_{2}\right]=0$. Let us consider point $\boldsymbol{\omega}_{1}$. We know $f_{t}\left(\boldsymbol{\omega}_{1}\right)=g_{11}\left(\boldsymbol{\omega}_{1}\right)=g_{12}\left(\boldsymbol{\omega}_{1}\right)=0$. Also $g_{2 i}\left(\boldsymbol{\omega}_{1}\right)=$ $\left(\boldsymbol{\omega}_{2} \times \boldsymbol{\omega}_{1}\right) \cdot\left(\mathbf{t}_{i} \times \boldsymbol{\omega}_{1}\right)$. Since both $\boldsymbol{\omega}_{1}$ and $\boldsymbol{\omega}_{2}$ lie on geodesic $n,\left(\boldsymbol{\omega}_{2} \times \boldsymbol{\omega}_{1}\right)$ is parallel to $\left(\mathbf{t}_{i} \times \boldsymbol{\omega}_{1}\right)$. Thus $g_{2 i}\left(\boldsymbol{\omega}_{1}\right)$ is zero only if $\left(\mathbf{t}_{i} \times \boldsymbol{\omega}_{1}\right)$ is zero. However, from (23) we have $\left(\mathbf{t}_{1} \times \mathbf{t}_{2}\right) \neq 0$, so either $g_{21}$ or $g_{22}$ is non-zero at $\boldsymbol{\omega}_{1}$.

If $g_{21}\left(\boldsymbol{\omega}_{1}\right) \neq 0$, then condition (15) cannot be satisfied and $\omega_{1}$ is a contradictory point. Again, it is not a singular point. The line tangent to $g_{11}$ at $\omega_{1}$ has direction $\boldsymbol{\omega}_{1} \times \mathbf{t}_{1}$ (and $\boldsymbol{\omega}_{1} \times \mathbf{t}_{1} \neq 0$, since $g_{21}\left(\boldsymbol{\omega}_{1}\right) \neq 0$ ), so $g_{11}$ is perpendicular to $n$ at this point. Since $f_{t}$ is identical to $n$, curves $g_{11}=0$ and $f_{t}=0$ create four areas around $\boldsymbol{\omega}_{1}$ with all possible sign combinations. Thus in one of the areas, condition (20) holds, and we obtain a contradictory area.

If $g_{22}\left(\boldsymbol{\omega}_{1}\right) \neq 0$, then condition (9) cannot be satisfied at $\boldsymbol{\omega}_{1}$. Again, at least one area around $\boldsymbol{\omega}_{1}$ is contradictory, since contour $g_{12}=0$ is perpendicular to $n$ at this point. This concludes the proof that if (23) is satisfied there exist contradictory areas on the half sphere. Section 4.2 discusses the case when (23) is not satisfied.

The rest of this section describes properties of the contradictory areas in order to provide a geometric intuition.

Just as we projected $\omega_{1}$ on geodesic $n$ connecting $\mathbf{t}_{1}$ and $\mathbf{t}_{2}$ to obtain $\mathbf{r}_{1}$, we project $\boldsymbol{\omega}_{2}$ on $n$ to obtain $\mathbf{r}_{2}$, and we project $\mathbf{t}_{1}$ and $\mathbf{t}_{2}$ on geodesic $l$, connecting $\boldsymbol{\omega}_{1}$ and $\boldsymbol{\omega}_{2}$, to obtain $\mathbf{r}_{3}$ and $\mathbf{r}_{4}$ (see Fig. 5). Point $\mathbf{r}_{2}$ is at the intersection of $f_{t}=0, g_{21}=0$, and $g_{22}=0 ; \mathbf{r}_{3}$ is at the intersection of $f_{\omega}=0, g_{11}=0$, and $g_{21}=0$; and $\mathbf{r}_{4}$ is at the intersection of $f_{\omega}=0$, $g_{12}=0$, and $g_{22}=0$. By the same argumentation as before, at each of the points we can choose two of the

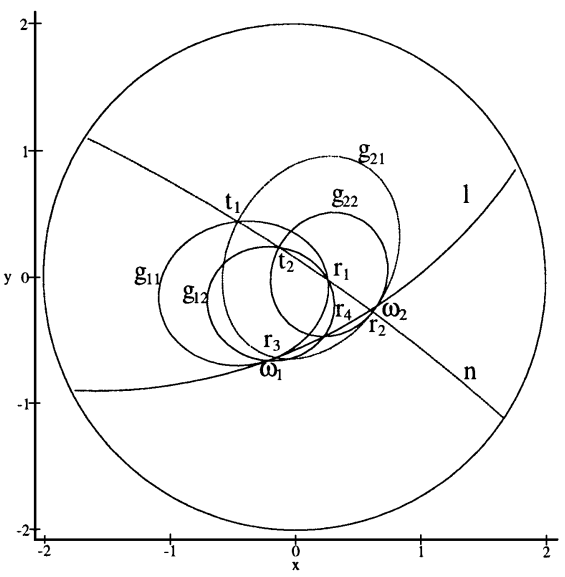

Figure 5. Separation of the sphere through curves $f_{i}=0$ and $g_{i j}=0$. Each of $\mathbf{t}_{1}, \mathbf{t}_{2}, \boldsymbol{\omega}_{1}, \boldsymbol{\omega}_{2}, \mathbf{r}_{1}, \mathbf{r}_{2}, \mathbf{r}_{3}$, and $\mathbf{r}_{4}$ lies at the intersection of three curves.

contours $f_{i}=0$ and $g_{i j}=0$ passing through the point and we obtain four areas of different sign combinations in the corresponding terms $f_{i}$ and $g_{i j}$ around the point; it can be shown that one of these areas is contradictory because one of the $\mathrm{CP}$-conditions is met.

The CP-conditions are constraints on the signs of the terms $f_{i}$ and $g_{i j}$. Thus the boundaries of the contradictory areas are formed by the curves $f_{i}=0$ and $g_{i j}=0$. As we have shown, the contradictory area and its boundaries must contain the points $\mathbf{r}_{1}, \mathbf{r}_{2}, \mathbf{r}_{3}$, and $\mathbf{r}_{4}$. For some motion configurations the boundaries might also contain $\mathbf{t}_{1}, \mathbf{t}_{2}, \boldsymbol{\omega}_{1}$, and $\boldsymbol{\omega}_{2}$. It can, however, be verified that no neighborhood around $\mathbf{t}_{1}, \mathbf{t}_{2}, \boldsymbol{\omega}_{1}$, and $\boldsymbol{\omega}_{2}$ needs to be contradictory. It can also be verified, by examining all the possibilities for the signs of terms $f_{i}$ and $g_{i j}$ in the CP-conditions, that points $\mathbf{t}_{1}, \mathbf{t}_{2}, \boldsymbol{\omega}_{1}$, and $\boldsymbol{\omega}_{2}$ cannot lie inside a contradictory area, since at least one of their neighboring areas is ambiguous. Figures 6 and 7 show the contradictory areas for both halves of the sphere for two different motion configurations.

Finally, let us consider the boundaries of the contradictory areas. As defined in Section 3, we allow the depths of the surfaces in view to take any value greater than zero (including infinity). Thus at any point $\mathbf{r}$ the motion vector $\dot{\mathbf{r}}$ could be in the direction of $v_{\text {rot }}(\mathbf{r})$, but not in the direction of $v_{\text {tr }}(\mathbf{r})$. This allows us to describe the depth values at possible boundaries of a contradictory area: At points on curve $f_{\omega}=0$ both $Z_{1}$ and $Z_{2}$ can be infinite, thus boundary points on this curve are not elements of the contradictory area. Boundary 


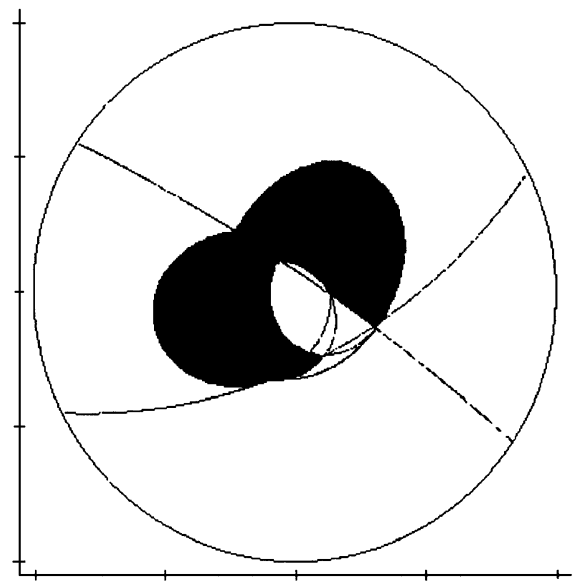

(a)

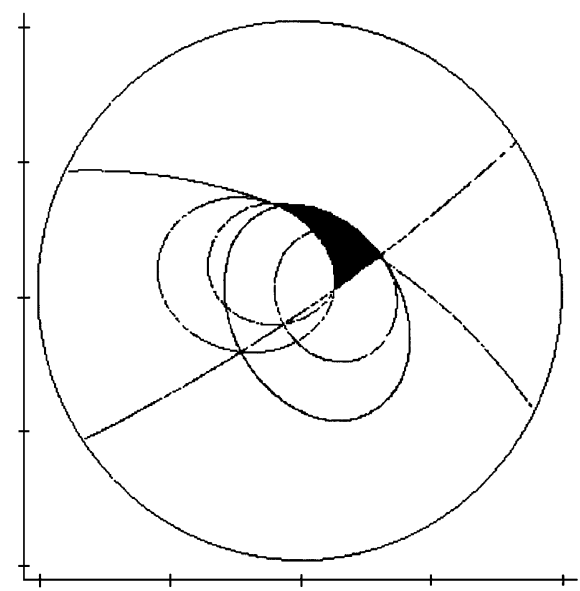

(b)

Figure 6. Contradictory areas for both halves of the sphere for the two motions shown in Fig. 5.

points on all other curves $\left(f_{t}=0\right.$, or $\left.g_{i j}=0\right)$ are contradictory, since one of the depths $Z_{1}$ and $Z_{2}$ would have to be zero.

\subsection{Half Sphere Image: The Ambiguous Case}

Let us now consider the case $\left(\mathbf{t}_{1} \times \mathbf{t}_{2}\right) \cdot\left(\boldsymbol{\omega}_{1} \times \boldsymbol{\omega}_{2}\right)=0$. However, we still assume $\mathbf{t}_{1} \times \mathbf{t}_{2} \neq 0$ and $\boldsymbol{\omega}_{1} \times \boldsymbol{\omega}_{2} \neq 0$. Then contour $f_{t}=0$ is perpendicular to $f_{\omega}=0$, the projections of $\boldsymbol{\omega}_{1}$ and $\boldsymbol{\omega}_{2}$ on $f_{t}=0$ and the projections of $\mathbf{t}_{1}$ and $\mathbf{t}_{2}$ on $f_{\omega}=0$ coincide in one point $\mathbf{r}_{1}$, i.e., $\mathbf{r}_{1}=\mathbf{r}_{2}=\mathbf{r}_{3}=\mathbf{r}_{4}$. Point $\mathbf{r}_{1}$ lies at the intersection of all six curves $f_{i}=0$ and $g_{i j}=0$. In this case there

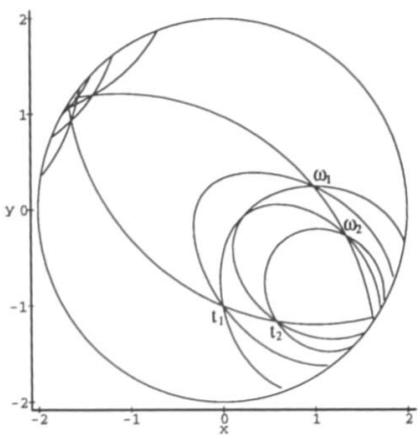

(a)

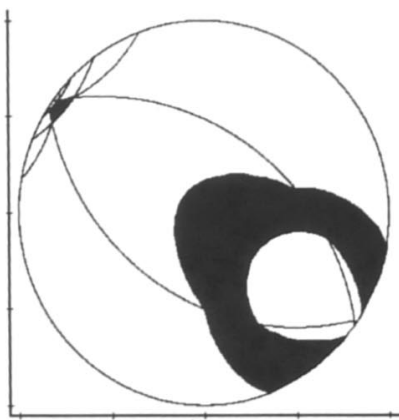

(b)

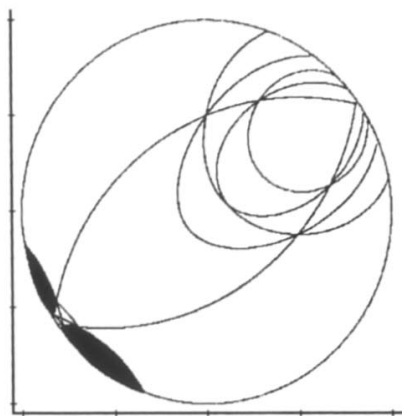

(c)

Figure 7. (a) Motion configuration. (b) and (c) Contradictory areas for both halves of the sphere.

could exist ambiguity, if certain additional conditions are satisfied.

First we investigate possible positions of points $\mathbf{t}_{1}, \mathbf{t}_{2}$, $\boldsymbol{\omega}_{1}$ and $\boldsymbol{\omega}_{2}$ on the hemisphere, bounded by equator $q$; then we describe additional conditions on the orientation of vectors $\mathbf{t}_{i}$ and $\boldsymbol{\omega}_{i}$ with respect to the hemisphere.

As shown in Section 3.5, two antipodal points $\mathbf{r}$ and $-\mathbf{r}$ can be ambiguous only if (22) holds. Thus if the border of the area defined by (22) intersects $q$, there will be a contradiction in the image. 
If curve $g_{i j}=0$ intersects $q$ at point $\mathbf{p}$, at least one of the areas around $\mathbf{p}$ does not satisfy condition (22). Unless $\mathbf{t}_{1}, \mathbf{t}_{2}, \boldsymbol{\omega}_{1}$ and $\boldsymbol{\omega}_{2}$ all are on the boundary of the hemisphere (and then the motions are not ambiguous), there is a contradictory area in the image (either around $\mathbf{p}$ or $-\mathbf{p})$.

By intersecting the zero motion contour $g_{i j}=0$ with the border $q$ of the hemisphere (see Appendix B), we find that contour $g_{i j}=0$ does not intersect the great circle $q$ if

$l=\left[\mathbf{t}_{j} \boldsymbol{\omega}_{i} \mathbf{n}_{0}\right]^{2}-4\left(\boldsymbol{\omega}_{i} \cdot \mathbf{n}_{0}\right)\left(\mathbf{t}_{j} \cdot \mathbf{n}_{0}\right)\left(\boldsymbol{\omega}_{i} \cdot \mathbf{t}_{j}\right)<0$

A half sphere contains, for each of the translation vectors $\mathbf{t}_{i}$ and the rotation vectors $\boldsymbol{\omega}_{i}$, exactly one of the vectors $+\mathbf{t}_{i}$ or $-\mathbf{t}_{i}$ and $+\boldsymbol{\omega}_{i}$ or $-\boldsymbol{\omega}_{i}$. Let us refer to the vectors in the considered hemisphere as $\tilde{\mathbf{t}}_{i}$ and $\tilde{\boldsymbol{\omega}}_{i}$. From Eq. (26), taking into account that $\left(\tilde{\mathbf{t}}_{i} \cdot \mathbf{n}_{0}\right) \geq 0$ and $\left(\tilde{\boldsymbol{\omega}}_{i} \cdot \mathbf{n}_{0}\right) \geq 0$, we see that $l>0$ when one of the following holds: if for any $\tilde{\boldsymbol{\omega}}_{i}, \tilde{\mathbf{t}}_{j},\left(\tilde{\boldsymbol{\omega}}_{i} \cdot \tilde{\mathbf{t}}_{j}\right)<0$ (i.e., $\tilde{\boldsymbol{\omega}}_{i}$ and $\tilde{\mathbf{t}}_{j}$ form an angle greater than $90^{\circ}$ ); or $\left(\tilde{\boldsymbol{\omega}}_{i} \cdot \tilde{\mathbf{t}}_{j}\right)>0$ and $\tilde{\boldsymbol{\omega}}_{i}$ and $\tilde{\mathbf{t}}_{j}$ are such that $\left[\mathbf{t}_{j} \boldsymbol{\omega}_{i} \mathbf{n}_{0}\right]^{2}>$ $4\left(\boldsymbol{\omega}_{i} \cdot \mathbf{n}_{0}\right)\left(\mathbf{t}_{j} \cdot \mathbf{n}_{0}\right)\left(\boldsymbol{\omega}_{i} \cdot \mathbf{t}_{j}\right)$ (i.e., $\tilde{\boldsymbol{\omega}}_{i}$ and $\tilde{\mathbf{t}}_{j}$ must be close to the border).

Any CP-condition depends on signs of three functions $f_{i}, g_{j j}$, and $g_{k l}=0$ (with $k \neq l$ ). The corresponding contours intersect only in $\mathbf{r}_{1}$ and one of the points $\mathbf{t}_{1}, \mathbf{t}_{2}, \boldsymbol{\omega}_{1}$, or $\boldsymbol{\omega}_{2}$. Since all the zero motion contours have to be closed curves on the hemisphere, we can conclude that if there exists a contradictory area, it also has to be in a neighborhood of $\mathbf{r}_{1}$.

It can be verified that, for a hemisphere to contain only ambiguous areas, the two translations have to have the same sign, that is $\operatorname{sgn}\left(\mathbf{t}_{1} \cdot \mathbf{n}_{0}\right)=\operatorname{sgn}\left(\mathbf{t}_{2} \cdot \mathbf{n}_{0}\right)$. Also the two rotations have to have the same sign, i.e., $\operatorname{sgn}\left(\boldsymbol{\omega}_{1} \cdot \mathbf{n}_{0}\right)=\operatorname{sgn}\left(\boldsymbol{\omega}_{2} \cdot \mathbf{n}_{0}\right)$. Furthermore, the relative positions of $\mathbf{t}_{1}, \mathbf{t}_{2}, \boldsymbol{\omega}_{1}$, and $\boldsymbol{\omega}_{2}$ have to be such that

$$
\begin{gathered}
\operatorname{sgn}\left(\left(\left(\boldsymbol{\omega}_{1} \times \boldsymbol{\omega}_{2}\right) \times\left(\mathbf{t}_{1} \times \mathbf{t}_{2}\right)\right) \cdot \mathbf{n}_{0}\right) \\
\quad=\operatorname{sgn}\left(\mathbf{t}_{1} \cdot \mathbf{n}_{0}\right) \operatorname{sgn}\left(\boldsymbol{\omega}_{1} \cdot \mathbf{n}_{0}\right)
\end{gathered}
$$

Intuitively this means, when rotating $f_{\omega}=0$ in the orientation given by the rotations in order to make $f_{\omega}=0$ and $f_{t}=0$ parallel, the order of points $\mathbf{t}_{1}$ and $\mathbf{t}_{2}$ on $f_{t}=0$ is opposite to the order of points $\tilde{\boldsymbol{\omega}}_{1}$ and $\tilde{\omega}_{2}$ on $f_{\omega}=0$ (moving in the same direction along $f_{\omega}=0$ and $\left.f_{t}=0\right)$, if $\operatorname{sgn}\left(\mathbf{t}_{1} \cdot \mathbf{n}_{0}\right)=1$. Otherwise, if $\operatorname{sgn}\left(\mathbf{t}_{1} \cdot \mathbf{n}_{0}\right)=-1$, then the order of points $-\mathbf{t}_{1}$ and $-\mathbf{t}_{2}$ on $f_{t}=0$ must be the same as the order of points $\tilde{\boldsymbol{\omega}}_{1}$ and $\tilde{\boldsymbol{\omega}}_{2}$ on $f_{\omega}=0$.
In summary, we have shown that two rigid motions could be ambiguous on one hemisphere, if $\mathbf{t}_{i} \neq 0$, $\boldsymbol{\omega}_{i} \neq 0$, and $\left(\mathbf{t}_{1} \times \mathbf{t}_{2}\right)$ is perpendicular to $\left(\boldsymbol{\omega}_{1} \times \boldsymbol{\omega}_{2}\right)$, but only if certain sign and certain distance conditions on $\mathbf{t}_{1}, \mathbf{t}_{2}, \boldsymbol{\omega}_{1}$ and $\boldsymbol{\omega}_{2}$ are met. In addition, as shown in Section 3, the two surfaces in view are constrained by a second- and a third-order surface (as shown in Eqs. (13) and (14)). Figure 8 gives an example of such a configuration.

In the next section we discuss the special cases and show that they do not allow for ambiguity. Thus the case of $\left(\mathbf{t}_{1} \times \mathbf{t}_{2}\right)$ being perpendicular to $\left(\boldsymbol{\omega}_{1} \times \boldsymbol{\omega}_{2}\right)$ is the only case where two motions can produce the same

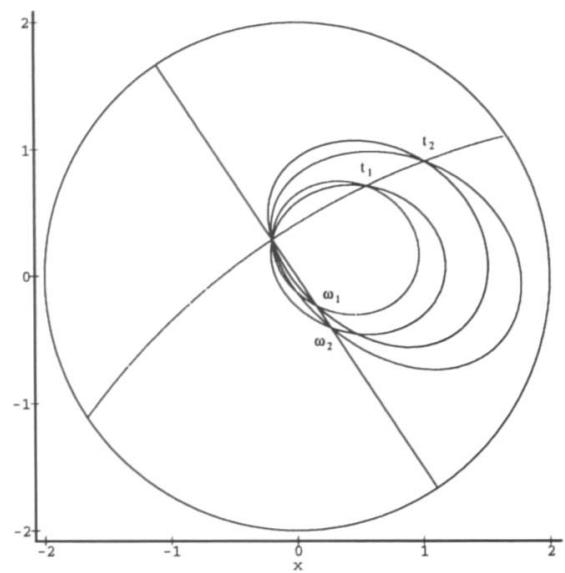

(a)

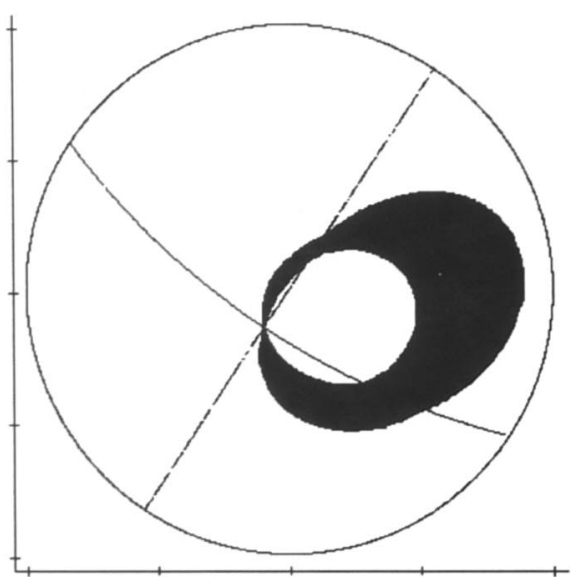

(b)

Figure 8. Both halves of the sphere showing two rigid motions for which there do not exist contradictory areas in one hemisphere. (a) Hemisphere containing only ambiguous areas. (b) Contradictory areas for the other hemisphere. 
direction of the motion field on a hemisphere. An analysis concerned with ambiguities due to more than two rigid motions is given in Section 6.

\section{Special Cases}

In previous sections, we assumed that $\mathbf{t}_{1} \times \mathbf{t}_{2} \neq 0$ and $\boldsymbol{\omega}_{1} \times \boldsymbol{\omega}_{2} \neq 0$. Here we show that if these conditions do not hold, then the two motions are not ambiguous.

In Section 3 we assumed all four vectors $\mathbf{t}_{1}, \mathbf{t}_{2}, \boldsymbol{\omega}_{1}$, and $\boldsymbol{\omega}_{2}$ to be of unit length. Here the four vectors can also be zero. Thus we have two different motions (i.e., $\mathbf{t}_{1} \neq \mathbf{t}_{2}$, or $\boldsymbol{\omega}_{1} \neq \boldsymbol{\omega}_{2}$ ), such that $\mathbf{t}_{1} \times \mathbf{t}_{2}=0$ and/or $\boldsymbol{\omega}_{1} \times \boldsymbol{\omega}_{2}=0$.

To cover all possible cases we are required to make a minor assumption about the depth $Z_{1}$ and $Z_{2}$ for the case where $\boldsymbol{\omega}_{1}=\boldsymbol{\omega}_{2}$.

Let $\boldsymbol{\omega}_{1}=\boldsymbol{\omega}_{2}$. Then we have $\mathbf{t}_{1} \neq \mathbf{t}_{2}$, and $f_{\omega}=0$ everywhere. From (10) we obtain the constraint $\operatorname{sgn}\left(\frac{1}{Z_{1}} g_{21}\right)=\operatorname{sgn}\left(\frac{1}{Z_{2}} g_{22}\right)$, so at points where $g_{21}$ and $g_{22}$ have different signs, the only possible solution is $1 / Z_{1}=1 / Z_{2}=0$. Infinite values for both depths in these areas would result in pure rotational flow fields in these areas and thus in an ambiguity. The same kind of ambiguity would occur if we considered the full flow. Therefore, it seems reasonable to assume that at least at one point in the areas where $g_{11} g_{22}<0$, both depths $Z_{1}$ and $Z_{2}$ are not infinite. Under this assumption there does not exist ambiguity for the case of $\boldsymbol{\omega}_{1}=\boldsymbol{\omega}_{2}$. In the following we thus assume $\boldsymbol{\omega}_{1} \neq \boldsymbol{\omega}_{2}$.

Next we provide a lemma that will be of use in the following proofs concerned with special cases as well as in the proof for full flow in Section 7.

Lemma 1. Let $\boldsymbol{\omega}_{i} \neq 0, \mathbf{t}_{j} \neq 0$. As in the previous section, let the image be a half sphere with equator $q$, let $\mathbf{n}_{0}$ be a unit vector normal to the plane of $q$. Then equation

$$
\operatorname{sgn}\left(\frac{1}{Z} f_{t}\right)=\sigma \operatorname{sgn}\left(g_{i j}\right)
$$

where $\sigma= \pm 1$, can be satisfied everywhere in the image only if $\mathbf{t}_{j} \times \mathbf{n}_{0}=0$ and $\boldsymbol{\omega}_{i} \cdot \mathbf{t}_{j}=0$.

Proof: Since $\boldsymbol{\omega}_{i}$ and $\mathbf{t}_{j}$ are non-zero, there are points in the image where $g_{i j} \neq 0$. Thus $\mathbf{t}_{1} \times \mathbf{t}_{2}$ must be nonzero and geodesic $n$ connecting $\mathbf{t}_{1}$ and $\mathbf{t}_{2}$ is well defined. Equation (28) can be satisfied only if the zero motion contour is degenerate, i.e., $\boldsymbol{\omega}_{i} \cdot \mathbf{t}_{j}=0$ (as in Fig. 13(b)).

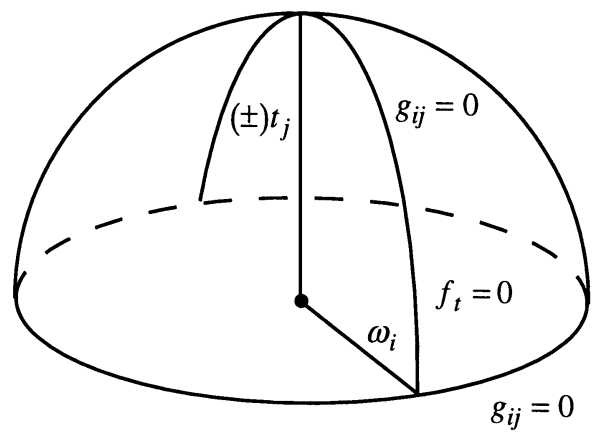

Figure 9. If $\operatorname{sgn}\left(\frac{1}{Z} f_{t}\right)=\sigma \operatorname{sgn}\left(g_{i j}\right)$ everywhere, the zero motion contour $g_{i j}=0$ consists of two great circles, one identical to the border of the hemisphere, the other identical to $f_{t}=0$.

Then the contour consists of two great circles. One of the circles must be identical to the geodesic $n$, and the other circle must be identical to $q$, the border of the image. This is possible only if $\mathbf{t}_{j} \times \mathbf{n}_{0}=0$ (see Fig. 9).

We now consider two special cases in parts A and B.

A: Let us assume that all $\mathbf{t}_{i}$ and $\boldsymbol{\omega}_{i}$ are non-zero.

If $\mathbf{t}_{1} \times \mathbf{t}_{2}=0$, then $f_{t}$ is zero everywhere. Thus from condition (9) we obtain $\operatorname{sgn}\left(g_{12}\right)=\operatorname{sgn}\left(g_{22}\right)$. Since all four vectors are non-zero, this is possible only if $\boldsymbol{\omega}_{1} \times \boldsymbol{\omega}_{2}=0$.

So we only need to consider the case $\boldsymbol{\omega}_{1} \times \boldsymbol{\omega}_{2}=0$, and since we also assume $\boldsymbol{\omega}_{1} \neq \boldsymbol{\omega}_{2}$, we have $\boldsymbol{\omega}_{2}=-\boldsymbol{\omega}_{1}$. Then at any point in the image, $g_{2 j}(\mathbf{r})=-g_{1 j}(\mathbf{r})$. Thus (9) can be satisfied only if $\operatorname{sgn}\left(\frac{1}{Z_{1}} f_{t}\right)=\operatorname{sgn}\left(g_{22}\right)$. According to the lemma, this is possible only if $\mathbf{t}_{2} \times \mathbf{n}_{0}=0$.

Similarly (15) can be satisfied only if $\operatorname{sgn}\left(\frac{1}{Z_{2}} f_{t}\right)=$ $\operatorname{sgn}\left(g_{21}\right)$. So from the lemma we obtain $\mathbf{t}_{1} \times \mathbf{n}_{0}=0$. Therefore, we have $\mathbf{t}_{1} \times \mathbf{t}_{2}=0$, function $f_{t}$ is zero everywhere, and the motions are contradictory.

B: If one of the motion parameters is zero, we obtain either a pure translational or a pure rotational flow field. By considering all the possible cases, it can be verified that the two motions are not ambiguous. Here we just consider one of the more difficult cases.

Let $\boldsymbol{\omega}_{1}=0, \boldsymbol{\omega}_{2} \neq 0, \mathbf{t}_{1} \neq 0$, and $\mathbf{t}_{2} \neq 0$. Then at any point, $g_{11}=g_{12}=f_{\omega}=0$. So from (9) we obtain $\operatorname{sgn}\left(\frac{1}{Z_{1}} f_{t}\right)=\operatorname{sgn}\left(g_{22}\right)$, from (15) we have $0=\operatorname{sgn}\left(-\frac{1}{Z_{2}} f_{t}+g_{21}\right)$, or $\operatorname{sgn}\left(\frac{1}{Z_{2}} f_{t}\right)=\operatorname{sgn}\left(g_{21}\right)$. From the lemma, this is possible only if $\mathbf{t}_{2} \times \mathbf{n}_{0}=0$ and 
$\mathbf{t}_{1} \times \mathbf{n}_{0}=0$; thus again we obtain $\mathbf{t}_{1} \times \mathbf{t}_{2}=0$ and the motions are contradictory.

If two of the motions are zero, that is if either $\mathbf{t}_{1}=$ $\mathbf{t}_{2}=0$ or $\mathbf{t}_{1}=\boldsymbol{\omega}_{2}=0$ (or equivalently $\mathbf{t}_{2}=\boldsymbol{\omega}_{1}=0$ ) we obtain either two rotational, or one translational and one rotational field, which obviously cannot have the same direction.

\section{Ambiguity of More than Two Motions}

Let us consider three different rigid motions $\left(\mathbf{t}_{1}, \boldsymbol{\omega}_{1}\right)$, $\left(\mathbf{t}_{2}, \boldsymbol{\omega}_{2}\right)$, and $\left(\mathbf{t}_{3}, \boldsymbol{\omega}_{3}\right)$, such that any two of the motions are ambiguous. Because we consider more motions now, we introduce subscripts and denote $f_{\omega i j}(\mathbf{r})=$ $\left[\boldsymbol{\omega}_{i} \boldsymbol{\omega}_{j} \mathbf{r}\right]$ and $f_{t i j}=\left[\mathbf{t}_{i} \mathbf{t}_{j} \mathbf{r}\right]$. The analysis is carried out similar to the case of two motions, by first developing conditions for points where one of the three depth maps has to be negative which are then used to show that almost all motion triplets can be distinguished. In the following we describe the conditions that must be met when the three motions are ambiguous.

\subsection{More Critical Surface Constraints}

As we have shown in Section 3, motions $\left(\mathbf{t}_{1}, \boldsymbol{\omega}_{1}\right)$ and $\left(\mathbf{t}_{3}, \boldsymbol{\omega}_{3}\right)$ can yield a compatible motion field only if the depth $1 / Z_{3}$ satisfies constraints given by a second- and a third-order surface. These surfaces can be written as $s_{31}=g_{31} / f_{t 13}$ and $s_{31}^{\prime}=-f_{\omega 13} / g_{13}$.

The depth $1 / Z_{3}$ must also satisfy constraints given by surfaces $s_{32}=g_{32} / f_{t 23}$ and $s_{32}^{\prime}=-f_{\omega 23} / g_{23}$. To find out whether all the constraints can be satisfied simultaneously, it is sufficient to check all the possible constraint pairs. Clearly, constraints $s_{31}$ and $s_{31}^{\prime}$ alone are not contradictory, and we can always find a solution to constraints $s_{32}$ and $s_{32}^{\prime}$. Thus we need to consider the four pairs $s_{31}$ and $s_{32}, s_{31}$ and $s_{32}^{\prime}, s_{31}^{\prime}$ and $s_{32}$, and $s_{31}^{\prime}$ and $s_{32}^{\prime}$.

Using the same method as in Section 3.4, we obtain the contradictory conditions

$$
\begin{aligned}
& \operatorname{sgn}\left(g_{11} f_{t 23}\right)=\operatorname{sgn}\left(g_{22} f_{t 31}\right)=\operatorname{sgn}\left(g_{33} f_{t 12}\right) \\
& \operatorname{sgn}\left(g_{11} f_{t 23}\right)=\operatorname{sgn}\left(g_{22} g_{13}\right)=-\operatorname{sgn}\left(g_{33} g_{12}\right) \\
& \operatorname{sgn}\left(g_{11} g_{23}\right)=\operatorname{sgn}\left(g_{22} f_{t 13}\right)=-\operatorname{sgn}\left(g_{33} g_{21}\right) \\
& \operatorname{sgn}\left(g_{11} g_{23}\right)=-\operatorname{sgn}\left(g_{22} g_{13}\right)=-\operatorname{sgn}\left(g_{33} f_{\omega 12}\right)
\end{aligned}
$$

If any of these constraints is satisfied, there is no solution for $1 / Z_{3}$ and the three motions are contradictory.
However, examination of all the possible cases reveals that if any of conditions (30), (31), or (32) is satisfied, condition (29) also must be satisfied and it is thus sufficient to use condition (29).

Similar contradictory conditions exist for depth maps $Z_{1}$ and $Z_{2}$. In both cases, we obtain one condition identical to (29) and three more conditions that can be satisfied only if (29) is satisfied. Thus condition (29) is necessary and sufficient for three motions to be contradictory.

\subsection{Simple Contradictory Situations}

There are several simple configurations that lead to a contradiction. Let $\mathbf{r}_{12}=\left(\boldsymbol{\omega}_{1} \times \boldsymbol{\omega}_{2}\right) \times\left(\mathbf{t}_{1} \times \mathbf{t}_{2}\right)$. Since motions $\left(\mathbf{t}_{1}, \boldsymbol{\omega}_{1}\right)$ and $\left(\mathbf{t}_{2}, \boldsymbol{\omega}_{2}\right)$ are ambiguous, curves $g_{11}, g_{12}, g_{21}, g_{22}, f_{\omega 12}$, and $f_{t 12}$ all pass through point $\mathbf{r}_{12}$ and vectors $v_{\mathrm{tr}_{1}}, v_{\mathrm{rot}_{1}}, v_{\mathrm{tr}_{2}}$, and $v_{\mathrm{rot}_{2}}$ are all parallel at that point. Similarly we can define points $\mathbf{r}_{13}$ and $\mathbf{r}_{23}$.

We present two configurations $\mathrm{A}$ and $\mathrm{B}$.

A: Let $g_{11}$ and $g_{22}$ intersect at point $\mathbf{p}, \mathbf{p} \neq \mathbf{r}_{12}$. Let the two zero motion contours cross at $\mathbf{p}$, and let $g_{33}(\mathbf{p}) \neq 0$. See Fig. 10 for an illustration.

Using the fact that geodesic $f_{t i j}$ intersects contour $g_{i i}$ only at points $\mathbf{r}_{i j}$ and $\mathbf{t}_{i}$, we obtain $f_{t 12}(\mathbf{p}) \neq 0$, $f_{t 31}(\mathbf{p}) \neq 0$, and $f_{t 23}(\mathbf{p}) \neq 0$. In a small neighborhood of point $\mathbf{p}$, quantities $g_{33}, f_{t 12}, f_{t 13}$, and $f_{t 23}$ do not change sign. Since $g_{11}$ and $g_{22}$ cross at $\mathbf{p}$, we obtain four neighborhood areas with all four sign combinations of $g_{11}$ and $g_{22}$. So we can always choose one neighborhood where condition (29) is satisfied.

B: Let contours $g_{11}$ and $g_{22}$ be tangent at point $\mathbf{r}_{12}$, and let $g_{33}\left(\mathbf{r}_{12}\right) \neq 0$.

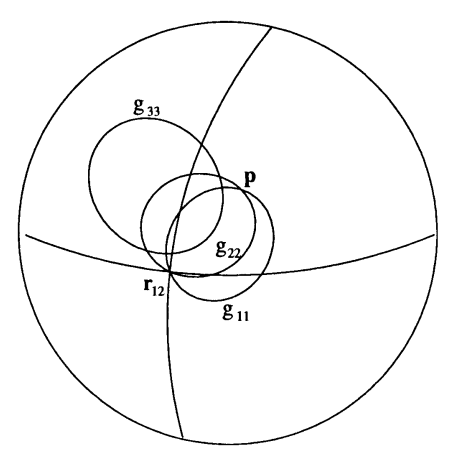

Figure 10. The zero motion contours $g_{11}$ and $g_{22}$ crossing at point $\mathbf{p}$. 
If contour $g_{11}$ or $g_{22}$ degenerates to a single point, then $\mathbf{r}_{12}=\mathbf{r}_{13}$ or $\mathbf{r}_{12}=\mathbf{r}_{23}$ and $g_{33}\left(\mathbf{r}_{12}\right)=0$. Therefore both $g_{11}$ and $g_{22}$ are non-degenerate and then $f_{t 31}\left(\mathbf{r}_{12}\right) \neq 0$ and $f_{t 23}\left(\mathbf{r}_{12}\right) \neq 0$, but $f_{t 12}\left(\mathbf{r}_{12}\right)=0$. Also, $g_{33}\left(\mathbf{r}_{12}\right) \neq 0$ implies $\left[\mathbf{t}_{1} \mathbf{t}_{2} \mathbf{t}_{3}\right] \neq 0$, that is $\mathbf{t}_{1}, \mathbf{t}_{2}$, and $\mathbf{t}_{3}$ do not all lie in the same plane.

Because the motions are pairwise ambiguous, the zero motion contours $g_{11}=0$ and $g_{22}=0$ must be closed curves on the image hemisphere. Thus areas inside and outside of $g_{11}$ and $g_{22}$ are well defined. We know $\operatorname{sgn}\left(\mathbf{t}_{1} \cdot \mathbf{n}_{0}\right)=\operatorname{sgn}\left(\mathbf{t}_{2} \cdot \mathbf{n}_{0}\right)$ and $\operatorname{sgn}\left(\boldsymbol{\omega}_{1} \cdot \mathbf{n}_{0}\right)=$ $\operatorname{sgn}\left(\boldsymbol{\omega}_{2} \cdot \mathbf{n}_{0}\right)$, therefore $\operatorname{sgn}\left(g_{11}\right)$ for points inside $g_{11}$ is the same as $\operatorname{sgn}\left(g_{22}\right)$ for points inside $g_{22}$.

If the two curvature vectors $k_{g_{11}}\left(\mathbf{r}_{12}\right)$ of $g_{11}=0$ and $k_{g_{22}}\left(\mathbf{r}_{12}\right)$ of $g_{22}=0$ have opposite sign (see Fig. 11(a)), we have $\operatorname{sgn}\left(f_{t 31}\left(\mathbf{r}_{12}\right)\right)=\operatorname{sgn}\left(f_{t 23}\left(\mathbf{r}_{12}\right)\right)$ (point $\mathbf{t}_{3}$ does not lie on $f_{t 12}=0$, and $\operatorname{sgn}\left(\mathbf{t}_{i} \cdot \mathbf{n}_{0}\right)$ is the same for all $i=1, \ldots, 3)$. In the two areas outside $g_{11}$ and outside $g_{22}, \operatorname{sgn}\left(g_{11} f_{t 23}\right)=\operatorname{sgn}\left(g_{22} f_{t 31}\right)$ is true.

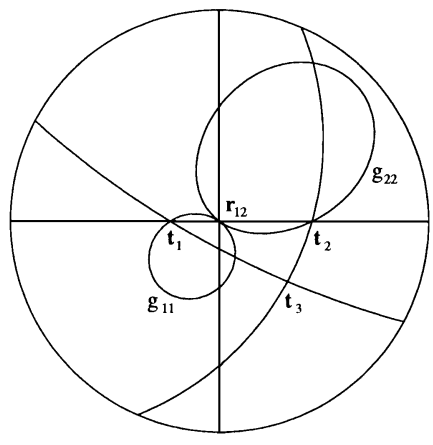

(a)

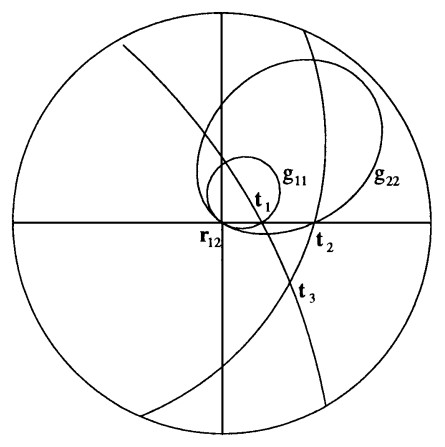

(b)

Figure 11. Two possible configurations when $g_{11}$ and $g_{22}$ are tangent at point $\mathbf{r}_{12}$. (a) The curvatures have opposite sign. (b) The curvatures have the same sign.
If the two curvature vectors $k_{g_{11}}\left(\mathbf{r}_{12}\right)$ and $k_{g_{22}}\left(\mathbf{r}_{12}\right)$ have the same sign (see Fig. 11(b)), $\operatorname{sgn}\left(f_{t 31}\left(\mathbf{r}_{12}\right)\right) \neq$ $\operatorname{sgn}\left(f_{t 23}\left(\mathbf{r}_{12}\right)\right)$. In the two areas where $\operatorname{sgn}\left(g_{11}\right) \neq$ $\operatorname{sgn}\left(g_{22}\right)$, again $\operatorname{sgn}\left(g_{11} f_{t 23}\right)$ and $\operatorname{sgn}\left(g_{22} f_{t 31}\right)$ are the same.

In both cases, there are two areas in the neighborhood of $\mathbf{r}_{12}$ where $\operatorname{sgn}\left(g_{11} f_{t 23}\right)=\operatorname{sgn}\left(g_{22} f_{t 31}\right)$ holds. Since $f_{t 12}$ is positive in one of the two areas and negative in the other, and $g_{33}$ does not change sign, condition (29) is satisfied in one of the areas.

\subsection{The Case $\left[\boldsymbol{t}_{1} \boldsymbol{t}_{2} \boldsymbol{t}_{3}\right] \neq 0$}

Configurations A and B above cover most of the cases that occur for three pairwise ambiguous motions. We now show that the motions are contradictory unless $\left[\mathbf{t}_{1}, \mathbf{t}_{2}, \mathbf{t}_{3}\right]=0$ or one of the zero motion contours degenerates to a single point.

We distinguish different cases using the number of intersection points of contours $g_{11}, g_{22}$, and $g_{33}$. For any $i \neq j$, let $m_{i j}$ be the number of intersection points of $g_{i i}$ and $g_{j j}$ where the two contours are not tangent. All three contours must intersect at such points (Section 6.2). We are using $m_{i j}$ instead of the number of all intersection points, because there are fewer cases to consider. Since both contours are of degree $2, m_{i j}$ can take on only the values 0,2 , or 4 .

I: Let $m_{12}=0$. Then contours $g_{11}$ and $g_{22}$ are tangent at point $\mathbf{r}_{12}$. So if $g_{33}\left(\mathbf{r}_{12}\right) \neq 0$, we have a contradiction (case B above). In order for $g_{33}\left(\mathbf{r}_{12}\right)$ to be zero, according to Appendix D, one of $g_{11}$ and $g_{22}$ has to degenerate to a point. Thus if $\boldsymbol{\omega}_{1} \times \mathbf{t}_{1}=0$ or $\boldsymbol{\omega}_{2} \times \mathbf{t}_{2}=0$, the motions are ambiguous. Otherwise we can find a contradiction.

II: Let $m_{12}=4$. This case is covered in Appendix E, which proves that the existence of four different intersection points is not possible. Thus the motions must be contradictory.

III: The only remaining case is $m_{12}=m_{13}=m_{23}$ $=2$. As shown in Appendix F, the motions are always contradictory in this case.

\subsection{Ambiguity of N Motions}

Let us now consider $N$ different motions. If two of the zero motion contours degenerate to a single point, the corresponding two motions are contradictory. Thus at most one of the zero motion contours degenerates to 


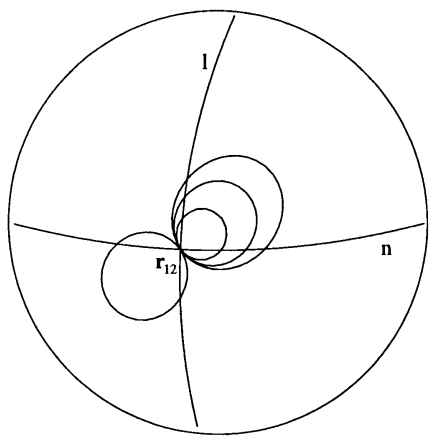

(a)

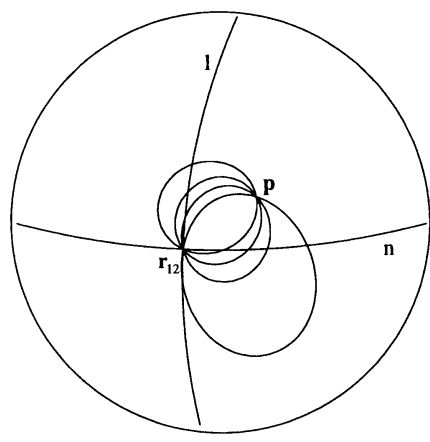

(b)

Figure 12. Two possible configurations of multiple ambiguous motions. (a) All the zero motion contours are tangent at point $\mathbf{r}_{12}$. (b) All the zero motion contours cross at point $\mathbf{p}$.

a point. We therefore obtain three possible configurations of $N$ motions that can yield an ambiguity:

- All points $\mathbf{t}_{i}, i=1, \ldots, N$ lie on a geodesic $n$, all $\boldsymbol{\omega}_{i}, i=1, \ldots, N$ lie on a geodesic $l$ perpendicular to $n$ and all the contours $g_{i i}$ are tangent at point $\mathbf{r}_{12}$ (see Fig. 12(a)). (The conditions for this to occur are described in Appendix C.)

- For $i=1, \ldots, N$, all points $\mathbf{t}_{i}$ lie on a geodesic $n$, all points $\boldsymbol{\omega}_{i}$ lie on a geodesic $l$ perpendicular to $n$ and all the contours $g_{i i}$ intersect at points $\mathbf{r}_{12}$ and $\mathbf{p}$ (see Fig. 12(b)).

- For $i=1, \ldots, N-1$, all points $\mathbf{t}_{i}$ lie on a geodesic $n$, all $\boldsymbol{\omega}_{i}$ lie on a geodesic $l$ perpendicular to $n$, contours $g_{i i}$ intersect in $\mathbf{r}_{12}$ and one point $\mathbf{p}$, and $\mathbf{t}_{N}=\boldsymbol{\omega}_{N}=\mathbf{p}$.

In all cases, for any $\mathbf{t}_{i}$ there exists at most one $\boldsymbol{\omega}_{i}$ satisfying all the constraints. Ambiguity is possible only if additional constraints are satisfied. Any two motions must be ambiguous (see Section 4.2 for details). In addition, the surfaces in view must satisfy all the surface constraints described in Section 3.

\section{Ambiguities of the Full Flow}

Next we investigate the question of whether there can be any ambiguities at all if we consider the complete flow. Horn has shown in (Horn, 1987) that two motions can produce ambiguous flow fields only if the observed surfaces are certain hyperboloids of one sheet. We show that if we also consider the depth positivity constraint and if the image is a half of the sphere, then any two different motions can be distinguished.

Let the image be a hemisphere bounded by equator $q$. Let $\mathbf{n}_{0}$ be a unit vector normal to the plane of $q$. As in (Horn, 1987), let us assume that a motion $\left(\mathbf{t}_{1}, \boldsymbol{\omega}_{1}\right)$ along with a depth map $Z_{1}$, and a motion $\left(\mathbf{t}_{2}, \boldsymbol{\omega}_{2}\right)$ along with a depth map $Z_{2}$, yield the same flow field. At each point we obtain a vector equation

$$
\begin{aligned}
& -\frac{1}{Z_{1}}\left(\mathbf{r} \times\left(\mathbf{t}_{1} \times \mathbf{r}\right)\right)-\omega_{1} \times \mathbf{r} \\
& \quad=-\frac{1}{Z_{2}}\left(\mathbf{r} \times\left(\mathbf{t}_{2} \times \mathbf{r}\right)\right)-\boldsymbol{\omega}_{2} \times \mathbf{r}
\end{aligned}
$$

Projecting on directions $\mathbf{t}_{1} \times \mathbf{r}$ and $\mathbf{t}_{2} \times \mathbf{r}$, we obtain equations for the two critical surfaces

$$
\begin{aligned}
& \frac{1}{Z_{1}}\left[\mathbf{t}_{1} \mathbf{t}_{2} \mathbf{r}\right]+(\delta \omega \times \mathbf{r}) \cdot\left(\mathbf{t}_{2} \times \mathbf{r}\right)=0 \\
& \frac{1}{Z_{2}}\left[\mathbf{t}_{1} \mathbf{t}_{2} \mathbf{r}\right]+(\delta \omega \times \mathbf{r}) \cdot\left(\mathbf{t}_{1} \times \mathbf{r}\right)=0
\end{aligned}
$$

where $\delta \boldsymbol{\omega}=\omega_{2}-\omega_{1}$.

If $\mathbf{t}_{1} \neq 0, \mathbf{t}_{2} \neq 0$, and $\delta \boldsymbol{\omega} \neq 0$, then according to the lemma in the previous section, these equations can be satisfied everywhere in the image only if $\delta \boldsymbol{\omega} \cdot \mathbf{t}_{1}=0$, $\mathbf{t}_{1} \times \mathbf{n}_{0}=0, \delta \boldsymbol{\omega} \cdot \mathbf{t}_{2}=0$, and $\mathbf{t}_{2} \times \mathbf{n}_{0}=0$. Thus we we obtain $\mathbf{t}_{1} \times \mathbf{t}_{2}=0$. (This case corresponds to Section 4.5 in (Horn, 1987), that is, to the case where both critical surfaces consist of intersecting planes.) Therefore, we are left only with special cases: Ambiguity can occur only if $\mathbf{t}_{1} \times \mathbf{t}_{2}=0$, or $\delta \boldsymbol{\omega}=0$.

If $\mathbf{t}_{1} \times \mathbf{t}_{2}=0$ and $\delta \boldsymbol{\omega} \neq 0$, from constraint (34) we get for any $\mathbf{r}$

$$
(\delta \boldsymbol{\omega} \times \mathbf{r}) \cdot\left(\mathbf{t}_{2} \times \mathbf{r}\right)=0
$$

Since $\delta \boldsymbol{\omega} \neq 0, \mathbf{t}_{2}$ must be zero. Similarly from constraint (35) we get $\mathbf{t}_{1}=0$. Thus we have a pair of rigid 
motions with different rotations and zero translations. Clearly these two motions are not ambiguous.

There is one special case left, $\delta \boldsymbol{\omega}=0$. At each point we get a vector equation

$$
-\frac{1}{Z_{1}}\left(\mathbf{r} \times\left(\mathbf{t}_{1} \times \mathbf{r}\right)\right)=-\frac{1}{Z_{2}}\left(\mathbf{r} \times\left(\mathbf{t}_{2} \times \mathbf{r}\right)\right)
$$

Since we have two different motions and $\delta \omega=0$, we know $\mathbf{t}_{1} \neq \mathbf{t}_{2}$. So the equation can be satisfied only when $1 / Z_{1}=1 / Z_{2}=0$ for all points not lying on geodesic $n$ passing through $\mathbf{t}_{1}$ and $\mathbf{t}_{2}$. If we do not allow infinite depth, the motions are not ambiguous.

Thus, we have shown that two different motions cannot give rise to the same optical flow field on an hemisphere. However, in the critical case, that is when $\left(\boldsymbol{\omega}_{1} \times \boldsymbol{\omega}_{2}\right) \cdot\left(\mathbf{t}_{1} \times \mathbf{t}_{2}\right)=0$, also $\delta \boldsymbol{\omega} \cdot \mathbf{t}_{1}=0$ and $\delta \boldsymbol{\omega} \cdot \mathbf{t}_{2}=0$, and each of the two zero-motion contours $(\delta \boldsymbol{\omega} \times \mathbf{r}) \cdot\left(\mathbf{t}_{2} \times \mathbf{r}\right)=0$ and $(\delta \boldsymbol{\omega} \times \mathbf{r}) \cdot\left(\mathbf{t}_{1} \times \mathbf{r}\right)=0$ degenerates to two great circles perpendicular to each other. In this case, the areas where negative depth has to occur are found only near the border of the hemisphere. The size of these area is related to the absolute position of the vectors $\mathbf{t}_{1}$ and $\mathbf{t}_{2}$. The closer the $\mathbf{t}_{i}$ are to the center of the image, and the closer the absolute distance of geodesic $\left[\mathbf{t}_{1} \mathbf{t}_{2} \mathbf{r}\right]=0$ to the center, the smaller the areas of negative depth near the border of the hemisphere. Thus, this analysis also proves that $3 \mathrm{D}$ rigid motions do not necessarily give rise to unique flow fields in the case of a limited field of view (less than a hemisphere) and it explains the potential confusion of translation and rotation.

\section{Conclusions}

In this paper, we have analyzed the amount of information inherent in the directions of rigid flow fields. We have shown that in almost all cases there is enough information to determine up to a multiplicative constant both the 3D-rotational and 3D-translational motion from a hemispherical image. Ambiguities can result only if the surface in view satisfies certain inequality and equality constraints. Furthermore, for two or more $3 \mathrm{D}$ motions to be compatible the translation vectors must lie on a geodesic perpendicular to the geodesic through the rotation vectors. With this analysis we have also shown that visual motion analysis does not necessarily require the intermediate computation of optical flow or exact correspondence. Instead, many dynamic vision problems might be solved with the use of more qualitative flow estimates if appropriate global constraints are found.

\section{Appendix A: Zero Motion Contours}

Let us consider the following question: What is the locus of points where the flow due to the given rigid motion can possibly be zero? As in (Fermüller and Aloimonos, 1996) we can show that such points are constrained to lie on a second-order curve on the sphere.

The flow at point $\mathbf{r}$ can be zero only if the rotational and translational components at $\mathbf{r}$ are parallel to each other. Let $\mathbf{t}$ and $\boldsymbol{\omega}$ be translational and rotational velocity of the observer. Then the flow at point $\mathbf{r}$ can be zero only if

$$
(\mathbf{r} \times(\mathbf{t} \times \mathbf{r})) \times(\boldsymbol{\omega} \times \mathbf{r})=0
$$

By simple vector manipulation, from (A1) we obtain

$$
((\boldsymbol{\omega} \times \mathbf{r}) \cdot(\mathbf{t} \times \mathbf{r})) \mathbf{r}=0
$$

Since $\mathbf{r} \neq 0$, the flow at point $\mathbf{r}$ can be zero only if

$$
\begin{aligned}
(\boldsymbol{\omega} \times \mathbf{r}) \cdot(\mathbf{t} \times \mathbf{r}) & =0 \\
\text { or } \quad \boldsymbol{\omega} \cdot \mathbf{t}-(\boldsymbol{\omega} \cdot \mathbf{r})(\mathbf{t} \cdot \mathbf{r}) & =0
\end{aligned}
$$

Equation (A3) describes a second-order curve on the sphere, which we will call the zero motion contour of the rigid motion $(\mathbf{t}, \boldsymbol{\omega})$. The zero-motion contour consists of two closed curves on the sphere. As shown in Fig. 13, if $(\boldsymbol{\omega} \cdot \mathbf{t})>0$, one of the curves contains $\mathbf{t}_{\mathbf{0}}=\frac{\mathbf{t}}{|\mathbf{t}|}$ and $\boldsymbol{\omega}_{0}=\frac{\omega}{|\boldsymbol{\omega}|}$ and one contains $-\mathbf{t}_{\mathbf{0}}$ and $-\boldsymbol{\omega}_{0}$; if $(\boldsymbol{\omega} \cdot \mathbf{t})=0$ the two curves become great circles, one orthogonal to $\mathbf{t}$, the other orthogonal to $\boldsymbol{\omega}$; if $(\boldsymbol{\omega} \cdot \mathbf{t})<0$ one of the two curves passes through $\mathbf{t}_{0}$ and $-\boldsymbol{\omega}_{0}$ and the other through $-\mathbf{t}_{0}$ and $\boldsymbol{\omega}_{0}$.

\section{Appendix B: Zero Motion Contour Crossing the Border of the Image}

Let the half sphere image be bounded by equator $q$, and let $\mathbf{n}_{0}$ be a unit vector normal to the plane of $q$. We would like to know whether the zero motion contour of motion $(\mathbf{t}, \boldsymbol{\omega})$ intersects equator $q$.

Let us choose a Cartesian coordinate system such that $\mathbf{n}_{0}=[0,0,1]$. Let $\mathbf{t}=\left[t_{x}, t_{y}, t_{z}\right]$ and $\boldsymbol{\omega}=$ $\left[\omega_{x}, \omega_{y}, \omega_{z}\right]$. Points on equator $q$ can be written as $[\cos \phi, \sin \phi, 0]$. Thus the zero motion contour $(\boldsymbol{\omega} \times \mathbf{r})$. 


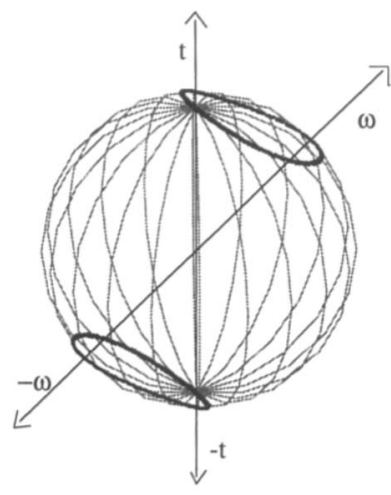

(a)

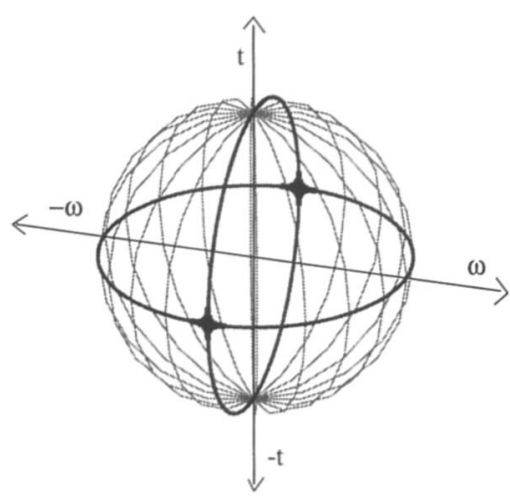

(b)

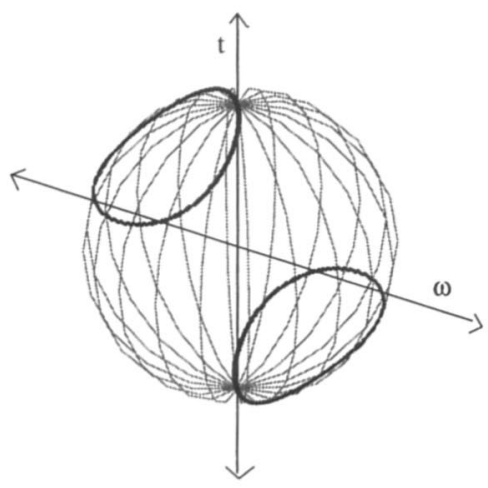

(c)

Figure 13. The zero motion contour (the locus of points $\mathbf{r}$ where $\dot{\mathbf{r}}$ could be zero) consists of two closed curves on the sphere. Three possible configurations are (a) $(\boldsymbol{\omega} \cdot \mathbf{t})>0$, (b) $(\boldsymbol{\omega} \cdot \mathbf{t})=0$, and (c) $(\boldsymbol{\omega} \cdot \mathbf{t})<0$.

$(\mathbf{t} \times \mathbf{r})=0$ intersects $q$ if equation

$$
\begin{aligned}
& \left(\omega_{x} t_{x}+\omega_{z} t_{z}\right) \sin ^{2} \phi \\
& \quad-\left(\omega_{y} t_{x}+\omega_{x} t_{y}\right) \sin \phi \cos \phi \\
& +\left(\omega_{y} t_{y}+\omega_{z} t_{z}\right) \cos ^{2} \phi=0
\end{aligned}
$$

has a solution.
Writing $\lambda=\tan \phi$, we obtain a quadratic equation

$$
\begin{aligned}
& \left(\omega_{x} t_{x}+\omega_{z} t_{z}\right) \lambda^{2}-\left(\omega_{y} t_{x}+\omega_{x} t_{y}\right) \lambda \\
& +\left(\omega_{y} t_{y}+\omega_{z} t_{z}\right)=0
\end{aligned}
$$

This equation has a real solution if

$$
\begin{aligned}
l= & \left(\omega_{y} t_{x}+\omega_{x} t_{y}\right)^{2} \\
& -4\left(\omega_{x} t_{x}+\omega_{z} t_{z}\right)\left(\omega_{y} t_{y}+\omega_{z} t_{z}\right) \geq 0
\end{aligned}
$$

After some manipulation, we obtain

$$
\begin{aligned}
l & =\left(\omega_{y} t_{x}-\omega_{x} t_{y}\right)^{2}-4\left(\omega_{z} t_{z}\right)\left(\omega_{x} t_{x}+\omega_{y} t_{y}+\omega_{z} t_{z}\right) \\
& =\left[\mathbf{t} \omega \mathbf{n}_{0}\right]^{2}-4\left(\boldsymbol{\omega} \cdot \mathbf{n}_{0}\right)\left(\mathbf{t} \cdot \mathbf{n}_{0}\right)(\boldsymbol{\omega} \cdot \mathbf{t}) \geq 0
\end{aligned}
$$

\section{Appendix C: Tangent Zero Motion Contours}

Let $\left(\mathbf{t}_{1}, \boldsymbol{\omega}_{1}\right)$ and $\left(\mathbf{t}_{2}, \boldsymbol{\omega}_{2}\right)$ be two ambiguous motions. Since ambiguity is possible only when $\boldsymbol{\omega}_{1} \times \boldsymbol{\omega}_{2} \neq 0$, $\mathbf{t}_{1} \times \mathbf{t}_{2} \neq 0$, and $\left(\boldsymbol{\omega}_{1} \times \boldsymbol{\omega}_{2}\right) \cdot\left(\mathbf{t}_{1} \times \mathbf{t}_{2}\right)=0$, we can choose a Cartesian coordinate system such that $\hat{X}=\left(\boldsymbol{\omega}_{1} \times \boldsymbol{\omega}_{2}\right) /\left|\boldsymbol{\omega}_{1} \times \boldsymbol{\omega}_{2}\right|, \hat{Y}=\left(\mathbf{t}_{1} \times \mathbf{t}_{2}\right) /\left|\mathbf{t}_{1} \times \mathbf{t}_{2}\right|$, and $\hat{Z}=\hat{X} \times \hat{Y}$.

In this coordinate system, we can write $\mathbf{t}_{1}=$ $\left[U_{1}, 0, W_{1}\right], \mathbf{t}_{2}=\left[U_{2}, 0, W_{2}\right], \boldsymbol{\omega}_{1}=\left[0, \beta_{1}, \gamma_{1}\right]$, and $\boldsymbol{\omega}_{2}=\left[0, \beta_{2}, \gamma_{2}\right]$. If $W_{1}$ were zero, we would have $\mathbf{t}_{1} \cdot \boldsymbol{\omega}_{1}=0$; thus the zero motion contour $g_{11}$ would be degenerate. This is not possible if the motions are ambiguous. Thus $W_{1} \neq 0$, and similarly $W_{2}, \gamma_{1}$, and $\gamma_{2}$ are non-zero.

Let us investigate when the contours are tangent at point $[0,0,1]$. We can write $g_{11}(\mathbf{r})=\left(\boldsymbol{\omega}_{1} \cdot \mathbf{t}_{1}\right)(\mathbf{r} \cdot \mathbf{r})$ $-\left(\boldsymbol{\omega}_{1} \cdot \mathbf{r}\right)\left(\mathbf{t}_{1} \cdot \mathbf{r}\right)$. The gradient of function $g_{11}\left(\frac{d g_{11}(\mathbf{r})}{d \mathbf{r}}\right)$ provides a vector normal to contour $g_{11}$ at point $\mathbf{r}$, which can be written as $-\left(\boldsymbol{\omega}_{1} \cdot \mathbf{r}\right) \mathbf{t}_{1}-\left(\mathbf{t}_{1} \cdot \mathbf{r}\right) \boldsymbol{\omega}_{1}$.

Therefore, the vector tangent to $g_{11}$ at $\mathbf{r}$ is $\left(\boldsymbol{\omega}_{1} \cdot \mathbf{r}\right)$ $\left(\mathbf{t}_{1} \times \mathbf{r}\right)+\left(\mathbf{t}_{1} \cdot \mathbf{r}\right)\left(\boldsymbol{\omega}_{1} \times \mathbf{r}\right)$. At point $[0,0,1]$, the tangent vector is $\mathbf{u}_{1}=\left[W_{1} \beta_{1},-U_{1} \gamma_{1}, 0\right]$. Similarly we obtain a tangent vector $\mathbf{u}_{2}=\left[W_{2} \beta_{2},-U_{2} \gamma_{2}, 0\right]$ to $g_{22}$ at $\mathbf{r}$.

The two contours are tangent only if $\mathbf{u}_{1} \times \mathbf{u}_{2}=0$, i.e.,

$$
\frac{U_{1}}{W_{1}} \frac{\beta_{2}}{\gamma_{2}}=\frac{U_{2}}{W_{2}} \frac{\beta_{1}}{\gamma_{1}}
$$

If $\mathbf{r}_{12}$ is the intersection point of $g_{11}$ and $g_{22}$, this can be written as

$$
\frac{\tan \angle\left(\mathbf{t}_{1}, \mathbf{r}_{12}\right)}{\tan \angle\left(\boldsymbol{\omega}_{1}, \mathbf{r}_{12}\right)}=\frac{\tan \angle\left(\mathbf{t}_{2}, \mathbf{r}_{12}\right)}{\tan \angle\left(\boldsymbol{\omega}_{2}, \mathbf{r}_{12}\right)}
$$

where $L(\cdot, \cdot)$ denotes the angle between two vectors. 


\section{Appendix D: Contour $g_{33}$ Passing Through $\mathbf{r}_{12}$}

Let the zero motion contours $g_{11}$ and $g_{22}$ be tangent at point $\mathbf{r}_{12}$ and let $g_{33}\left(\mathbf{r}_{12}\right)=0$. We use the same coordinate system as in Appendix C. Since we can re-scale the vectors $\mathbf{t}_{i}, \boldsymbol{\omega}_{i}$, let $\mathbf{t}_{1}=\left[U_{1}, 0,1\right]$, $\boldsymbol{\omega}_{1}=\left[0, \beta_{1}, 1\right], \mathbf{t}_{2}=\left[U_{2}, 0,1\right], \boldsymbol{\omega}_{2}=\left[0, \beta_{2}, 1\right]$. In addition let $\mathbf{t}_{3}=\left[U_{3}, V_{3}, 1\right]$ and $\boldsymbol{\omega}_{3}=\left[\alpha_{3}, \beta_{3}, 1\right]$.

Since $g_{11}$ and $g_{22}$ are tangent, $U_{1} \beta_{2}=U_{2} \beta_{1}$. From $g_{33}\left(\mathbf{r}_{12}\right)=0$ we obtain $V_{3} \beta_{3}+\alpha_{3} U_{3}=0$. Motions $\left(\mathbf{t}_{1}, \boldsymbol{\omega}_{1}\right)$ and $\left(\mathbf{t}_{3}, \boldsymbol{\omega}_{3}\right)$ are ambiguous, so $\left(\mathbf{t}_{1} \times \mathbf{t}_{3}\right)$. $\left(\boldsymbol{\omega}_{1} \times \boldsymbol{\omega}_{3}\right)=0$, which can be written as $\left(V_{3} \beta_{1}+1\right)$ $\left(U_{1} \alpha_{3}+1\right)=1$. Similarly we have $\left(V_{3} \beta_{2}+1\right)$ $\left(U_{2} \alpha_{3}+1\right)=1$.

If $U_{1}=0$, then also $U_{2} \beta_{1}=0$. Since $U_{2} \neq U_{1}$, we obtain $\beta_{1}=0$, i.e., $\mathbf{t}_{1} \times \boldsymbol{\omega}_{1}=0$ and $g_{11}$ degenerates to a single point.

Similarly, if $U_{2}=0$, contour $g_{22}$ degenerates to a single point. Also $\beta_{1}=0$ or $\beta_{2}=0$ lead to degenerate zero motion contours.

If $U_{1}, U_{2}, \beta_{1}$, and $\beta_{2}$ are non-zero, we can compute $\alpha_{3}=-V_{3} \beta_{1} /\left(U_{1}\left(V_{3} \beta_{1}+1\right)\right)$ and $\alpha_{3}=$ $-V_{3} \beta_{2} /\left(U_{2}\left(V_{3} \beta_{2}+1\right)\right)$. Using $\beta_{1} / U_{1}=\beta_{2} / U_{2}$, we obtain

$$
\frac{V_{3}}{V_{3} \beta_{1}+1}=\frac{V_{3}}{V_{3} \beta_{2}+1}
$$

Since $\beta_{1} \neq \beta_{2}$, this equation can be satisfied only if $V_{3}=0$; but then $\left[\mathbf{t}_{1} \mathbf{t}_{2} \mathbf{t}_{3}\right]=0$.

In summary, if $g_{11}$ is tangent to $g_{22}$ at point $\mathbf{r}_{12}$ and $g_{33}\left(\mathbf{r}_{12}\right)=0$, then either one of the zero motion contours degenerates to a single point, or $\left[\mathbf{t}_{1} \mathbf{t}_{2} \mathbf{t}_{3}\right]=0$.

\section{Appendix E: The Case $m_{12}=4$ : Intersections of Three Zero Motion Contours}

For the case of $m_{12}=4$ the motions must be contradictory. The proof is carried out by showing that if $\left[\mathbf{t}_{1} \mathbf{t}_{2} \mathbf{t}_{3}\right] \neq 0$ there cannot exist more than three different intersection points of the zero motion contours $g_{11}$ and $g_{22}$.

Let point $\mathbf{p}$ lie on all three zero motion contours $g_{i i}$ for $i=1, \ldots, 3$. We show that if the motions are pairwise ambiguous, then either $\mathbf{p} \in\left\{\mathbf{r}_{12}, \mathbf{r}_{13}, \mathbf{r}_{23}\right\}$, or $\left[\mathbf{t}_{1} \mathbf{t}_{2} \mathbf{t}_{3}\right]=0$.

Let us choose a Cartesian coordinate system such that $\mathbf{p}=[0,0,1], \mathbf{t}_{1}=\left[U_{1}, 0,1\right]$, and $\boldsymbol{\omega}_{1}=$ $\left[0, \beta_{1}, 1\right]$ (we can re-scale vectors $\mathbf{t}_{i}$ and $\boldsymbol{\omega}_{i}$ ). Let $\mathbf{t}_{2}=\left[U_{2}, V_{2}, 1\right], \boldsymbol{\omega}_{2}=\left[\alpha_{2}, \beta_{2}, 1\right], \mathbf{t}_{3}=\left[U_{3}, V_{3}, 1\right]$, and $\boldsymbol{\omega}_{3}=\left[\alpha_{3}, \beta_{3}, 1\right]$.
Contours $g_{22}$ and $g_{33}$ pass through $\mathbf{p}$, so

$$
U_{2} \alpha_{2}+V_{2} \beta_{2}=0
$$

and

$$
U_{3} \alpha_{3}+V_{3} \beta_{3}=0
$$

Also, the motions are pairwise ambiguous, thus $\left(\boldsymbol{\omega}_{i} \times \boldsymbol{\omega}_{j}\right) \cdot\left(\mathbf{t}_{i} \times \mathbf{t}_{j}\right)=0$. Using (E1) and (E2), we obtain

$$
\left(U_{1} \alpha_{2}+1\right)\left(V_{2} \beta_{1}+1\right)=1
$$

and

$$
\left(U_{1} \alpha_{3}+1\right)\left(V_{3} \beta_{1}+1\right)=1
$$

There are several special cases we need to describe first.

If $V_{2}=0$, from (E1) we have $U_{2} \alpha_{2}=0$ and Eq. (E3) becomes $U_{1} \alpha_{2}=0$. Thus if $V_{2}=0$ and $\alpha_{2} \neq 0$, we obtain $U_{1}=U_{2}=0$. This is not possible since the motions are pairwise ambiguous and $\mathbf{t}_{1} \neq \mathbf{t}_{2}$. If $V_{2}=0$ and $\alpha_{2}=0$, then $\mathbf{p}=\mathbf{r}_{12}$.

Similarly, if $V_{3}=0$, then $\mathbf{p}=\mathbf{r}_{13}$.

If $U_{1}=0$, then $V_{2} \beta_{1}=0$ from (E3). We have already described the case $V_{2}=0$. If $U_{1}=0$ and $\beta_{1}=0$, then $\mathbf{t}_{1} \times \boldsymbol{\omega}_{1}=0$, contour $g_{11}$ degenerates to a single point, and $\mathbf{p}=\mathbf{r}_{12}=\mathbf{r}_{13}$.

From now on, let us assume that $U_{1}, V_{2}$, and $V_{3}$ are non-zero. Then we can compute $\beta_{2}$ and $\beta_{3}$ from (E1) and (E2). Since Eqs. (E3) and (E4) must be satisfied, $V_{2} \beta_{1}+1 \neq 0$ and $V_{3} \beta_{1}+1 \neq 0$, so we can also compute $\alpha_{2}$ and $\alpha_{3}$.

Now we can express $\left(\boldsymbol{\omega}_{2} \times \boldsymbol{\omega}_{3}\right) \cdot\left(\mathbf{t}_{2} \times \mathbf{t}_{3}\right)=0$ as

$$
\frac{\beta_{1}^{2}\left(U_{2} V_{3}-V_{2} U_{3}\right)\left(V_{3}\left(U_{2}-U_{1}\right)+V_{2}\left(U_{1}-U_{3}\right)\right)}{U_{1}^{2}\left(V_{2} \beta_{1}+1\right)\left(V_{3} \beta_{1}+1\right)}=0
$$

If $\beta_{1}=0$, then $U_{1} \alpha_{2}=0$. Since we assume $U_{1} \neq 0$, we have $\alpha_{2}=0$. Then also $V_{2} \beta_{2}=0$ and $V_{2} \neq 0$, so $\beta_{1}=\beta_{2}=\alpha_{2}=0$. This is impossible, since the motions would not be ambiguous.

If $\left(U_{2} V_{3}-V_{2} U_{3}\right)=0$, from (E1) and (E2) we obtain $\beta_{2} \alpha_{3}-\beta_{3} \alpha_{2}=0$. Then it can be verified that $\mathbf{p}=\mathbf{r}_{23}$.

Simple computation shows that $\left[\mathbf{t}_{1} \mathbf{t}_{2} \mathbf{t}_{3}\right]=$ $V_{3}\left(U_{2}-U_{1}\right)+V_{2}\left(U_{1}-U_{3}\right)$. Thus if $V_{3}\left(U_{2}-U_{1}\right)+$ $V_{2}\left(U_{1}-U_{3}\right)=0$, then $\left[\mathbf{t}_{1} \mathbf{t}_{2} \mathbf{t}_{3}\right]=0$.

Therefore, in all cases either $\mathbf{p} \in\left\{\mathbf{r}_{12}, \mathbf{r}_{13}, \mathbf{r}_{23}\right\}$, or $\left[\mathbf{t}_{1} \mathbf{t}_{2} \mathbf{t}_{3}\right]=0$. Since, however, $m_{12}=4$, it follows that the three motions cannot be ambiguous. 


\section{Appendix F: The Case $m_{i j}=2$}

Let us assume that $\left[\mathbf{t}_{1} \mathbf{t}_{2} \mathbf{t}_{3}\right] \neq 0$ and $m_{12}=m_{13}=$ $m_{23}=2$. In this case, there exist points $\mathbf{p}_{12} \neq \mathbf{r}_{12}$, $\mathbf{p}_{13} \neq \mathbf{r}_{13}$, and $\mathbf{p}_{23} \neq \mathbf{r}_{23}$. The corresponding zero motion contours cross at these points.

First we show that points $\mathbf{r}_{12}, \mathbf{r}_{13}$, and $\mathbf{r}_{23}$ all have to be different. Let us by contradiction assume that $\mathbf{r}_{12}=\mathbf{r}_{13}$. We know $\left[\mathbf{t}_{1} \mathbf{t}_{2} \mathbf{r}_{12}\right]=0$ and $\left[\mathbf{t}_{1} \mathbf{t}_{3} \mathbf{r}_{13}\right]=0$. Thus if $\mathbf{t}_{1} \neq \mathbf{r}_{12}$, then $\left[\mathbf{t}_{1} \mathbf{t}_{2} \mathbf{t}_{3}\right]=0$. Therefore, $\mathbf{t}_{1}=$ $\mathbf{r}_{12}=\mathbf{r}_{13}$.

Then also $\left[\boldsymbol{\omega}_{1} \boldsymbol{\omega}_{2} \mathbf{t}_{1}\right]=0$ and $\left[\boldsymbol{\omega}_{1} \boldsymbol{\omega}_{3} \mathbf{t}_{1}\right]=0$. Since contour $g_{11}$ is not a single point, we know $\mathbf{t}_{1} \times \boldsymbol{\omega}_{1} \neq 0$. Thus we obtain $\left[\boldsymbol{\omega}_{1} \boldsymbol{\omega}_{2} \boldsymbol{\omega}_{3}\right]=0$. Then both $\mathbf{t}_{2}$ and $\mathbf{t}_{3}$ lie on a geodesic that is perpendicular to the geodesic containing all $\boldsymbol{\omega}_{i}$ and passes through $\mathbf{t}_{1}$. Therefore, $\left[\mathbf{t}_{1} \mathbf{t}_{2} \mathbf{t}_{3}\right]=0$. Since this is a contradiction, $\mathbf{r}_{12} \neq \mathbf{r}_{13}$.

Clearly, the motions are contradictory unless $\left\{\mathbf{p}_{12}, \mathbf{p}_{13}, \mathbf{p}_{23}\right\} \subseteq\left\{\mathbf{r}_{12}, \mathbf{r}_{13}, \mathbf{r}_{23}\right\}$. Since $\mathbf{p}_{12} \neq \mathbf{r}_{12}$, without loss of generality we can assume $\mathbf{p}_{12}=\mathbf{r}_{13}$. Let us consider two possibilities.

If $g_{11}\left(\mathbf{r}_{23}\right)=0$, then points $\mathbf{r}_{12}, \mathbf{r}_{13}=\mathbf{p}_{12}$, and $\mathbf{r}_{23}$ all belong to $g_{11} \cap g_{22}$.

If $g_{11}\left(\mathbf{r}_{23}\right) \neq 0$, then $\mathbf{p}_{13} \neq \mathbf{r}_{23}\left(\right.$ since $g_{11}\left(\mathbf{p}_{13}\right)=0$ ). Also $\mathbf{p}_{13} \neq \mathbf{r}_{13}$, thus $\mathbf{p}_{13}=\mathbf{r}_{12}$. Then points $\mathbf{r}_{12}=\mathbf{p}_{13}$, $\mathbf{r}_{13}=\mathbf{p}_{12}$, and $\mathbf{r}_{23}$ all lie in $g_{22} \cap g_{33}$.

In both cases, three different points $\mathbf{r}_{12}, \mathbf{r}_{13}$, and $\mathbf{r}_{23}$ lie on two of the zero motion contours. Without loss of generality, we can assume these points belong to $g_{11} \cap g_{22}$. Since $m_{12}=2$, the two contours have at most 3 intersection points. Thus, the two contours must be tangent at one of the intersection points $g_{11} \cap g_{22}=$ $\left\{\mathbf{r}_{12}, \mathbf{r}_{13}, \mathbf{r}_{23}\right\}$.

If $g_{11}$ and $g_{22}$ are tangent at point $\mathbf{r}_{12}$ and $g_{33}\left(\mathbf{r}_{12}\right) \neq$ 0 , the motions are contradictory (case B of Section 6.2). If $g_{33}\left(\mathbf{r}_{12}\right)=0$, then either $\left[\mathbf{t}_{1} \mathbf{t}_{2} \mathbf{t}_{3}\right]=0$, or one of $g_{11}$, $g_{22}$ must degenerate to a single point (see Appendix D). Since this is not possible, the two contours must be tangent at point $\mathbf{q} \neq \mathbf{r}_{12}$. Without loss of generality, we can assume $\mathbf{q}=\mathbf{r}_{23}$.

Because none of the zero motion contours degenerates to a single point and because $\left[\mathbf{t}_{1} \mathbf{t}_{2} \mathbf{t}_{3}\right] \neq 0$, contour $g_{33}$ cannot be tangent to $g_{22}$ at point $\mathbf{q}=\mathbf{r}_{23}$ (see Appendix D).

Since point $\mathbf{q}$ lies on both $g_{11}$ and $g_{22}$, if $\mathbf{t}_{3}=\mathbf{q}$, then the motions can be pairwise ambiguous only if also $\boldsymbol{\omega}_{3}=\mathbf{q}$. But then $g_{33}$ degenerates to a single point.

Thus $\mathbf{t}_{3} \neq \mathbf{q}$. We know $\left[\mathbf{t}_{2} \mathbf{t}_{3} \mathbf{q}\right]=0$. If also $\left[\mathbf{t}_{1} \mathbf{t}_{3} \mathbf{q}\right]=0$, we obtain $\left[\mathbf{t}_{1} \mathbf{t}_{2} \mathbf{t}_{3}\right]=0$, which is a contradiction. So $f_{t 13}(\mathbf{q}) \neq 0$.

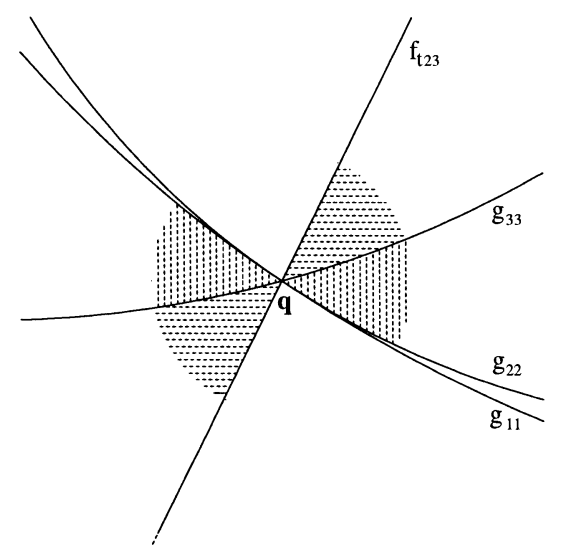

Figure 14. Configuration of the contours at point q. One of the shaded areas is contradictory.

Contours $g_{11}$ and $g_{22}$ can intersect at point $\mathbf{t}_{2}$ only if $\mathbf{t}_{2}=\mathbf{r}_{12}$. Since $\mathbf{q}=\mathbf{r}_{23} \neq \mathbf{r}_{12}$, we also have $\mathbf{q} \neq \mathbf{t}_{2}$. If $\left[\mathbf{t}_{1} \mathbf{t}_{2} \mathbf{q}\right]=0$, we again obtain $\left[\mathbf{t}_{1} \mathbf{t}_{2} \mathbf{t}_{3}\right]=0$. Therefore, $f_{t 12}(\mathbf{q}) \neq 0$.

Since $\mathbf{q}=\mathbf{r}_{23} \neq \mathbf{t}_{3}$, geodesic $f_{t 23}$ is not tangent to $g_{33}$ at $\mathbf{q}$. Also, since $\mathbf{q} \neq \mathbf{t}_{2}$, geodesic $f_{t 23}$ is not tangent to $g_{22}$ at $\mathbf{q}$.

In a small neighborhood of point $\mathbf{q}$, let us consider the four areas next to the contour $g_{33}$ (see Fig. 14). Since $g_{33}$ is crossing $g_{11}$ and $g_{22}$, the sign of $g_{11} g_{22}$ remains the same in all these areas.

Since $g_{33}$ is not tangent to $f_{t 23}$, we obtain all four sign combinations of $g_{33}$ and $f_{t 23}$ in these areas. Thus we can pick an area where both $\operatorname{sgn}\left(g_{11} f_{t 23}\right)=$ $\operatorname{sgn}\left(g_{22} f_{t 31}\right)$ and $\operatorname{sgn}\left(g_{33} f_{t 12}\right)=\operatorname{sgn}\left(g_{22} f_{t 31}\right)$ are satisfied. This area is then contradictory.

To summarize, if $\left[\mathbf{t}_{1} \mathbf{t}_{2} \mathbf{t}_{3}\right] \neq 0$ and $m_{12}=m_{13}=$ $m_{23}=2$, then the three motions are contradictory.

\section{References}

Ancona, N. and Poggio, T. 1995. Optical flow from 1-D correlation: Application to a simple time-to-crash detector. International Journal of Computer Vision, 14:131-146, Special Issue on Qualitative Vision, Y. Aloimonos (Ed.).

Faugeras, O.D., Lustman, F., and Toscani, G. 1987. Motion and structure from motion from point and line matches. In Proc. International Conference on Computer Vision, pp. 25-34.

Fermüller, C. 1995. Passive navigation as a pattern recognition problem. International Journal of Computer Vision, 14:147-158.

Fermüller, C. and Aloimonos, Y. 1995. Qualitative egomotion. International Journal of Computer Vision, 15:7-29.

Fermüller, C. and Aloimonos, Y. 1997. On the geometry of visual correspondence. International Journal of Computer Vision, 21(3), 223-247. 
Horn, B.K.P. 1987. Motion fields are hardly ever ambiguous. International Journal of Computer Vision, 1:259-274.

Horn, B.K.P. 1990. Relative orientation. International Journal of Computer Vision, 4:59-78.

Longuet-Higgins, H.C. 1981. A computer algorithm for reconstructing a scene from two projections. Nature, 293:133-135.

Maybank, S. 1993. Theory of Reconstruction from Image Motion, Springer: Berlin.

Negahdaripour, S. 1989. Critical surface pairs and triplets. International Journal of Computer Vision, 3:293-312.

Negahdaripour, S. and Horn, B.K.P. 1987. Direct passive navigation. IEEE Transactions on Pattern Analysis and Machine Intelligence, 9:163-176.
Spetsakis, M.E. and Aloimonos, J. 1990. Structure from motion using line correspondences. International Journal of Computer Vision, 4:171-183.

Tistarelli, M. and Sandini, G. 1992. Dynamic aspects in active vision. CVGIP: Image Understanding: Special Issue on Purposive, Qualitative, Active Vision, 56:108-129.

Tsai, R.Y. and Huang, T.S. 1984. Uniqueness and estimation of threedimensional motion parameters of rigid objects with curved surfaces. IEEE Transactions on Pattern Analysis and Machine Intelligence, 6:13-27.

Vieville, T. and Faugeras, O.D. 1994. Robust and fast computation of edge characteristics in image sequences. International Journal of Computer Vision, 13:153-179. 